\title{
CONCERNING CELLULAR DECOMPOSITIONS OF 3-MANIFOLDS THAT YIELD 3-MANIFOLDS
}

\author{
BY \\ STEVE ARMENTROUT( $\left.{ }^{1}\right)$
}

1. Introduction. In this paper, we shall study cellular decompositions of 3manifolds for which the associated decomposition space is also a 3-manifold. In [4], Bing raised the following question: Does each point-like decomposition of $E^{3}$ yield $E^{3}$ if it yields a 3-manifold? This question can be generalized as follows: If a cellular decomposition of a 3-manifold $M$ yields a 3-manifold $N$, then are $M$ and $N$ homeomorphic? We shall establish conditions sufficient to insure that, under the hypothesis stated, $M$ and $N$ are homeomorphic.

The main theorem of this paper can be stated as follows. Suppose that $M$ is a connected 3-manifold and $G$ is a cellular decomposition of $M$ such that the associated decomposition space is a 3-manifold $N$. Let $P$ denote the projection map from $M$ onto $N$, and let $H_{G}$ denote the union of all the nondegenerate elements of $G$. Thus $\mathrm{Cl} P\left[H_{G}\right]$ is the set of singular points of the map $P$. Our main result then states that if $N$ has a triangulation $T$ such that (1) no vertex of $T$ belongs to $\mathrm{Cl} P\left[H_{G}\right]$ and (2) for each 3-simplex $\sigma$ of $T, P^{-1}[\sigma]$ lies in an open 3-cell in $M$, then $M$ and $N$ are homeomorphic.

As a corollary of the main result, we obtain the following result concerning $E^{3}$. If $G$ is a point-like decomposition of $E^{3}$ such that $P\left[H_{G}\right]$ is nowhere dense and the associated decomposition space is a 3-manifold, then the decomposition space is homeomorphic to $E^{3}$. In particular, if $G$ is a point-like decomposition of $E^{3}$ such that $\dot{P}\left[H_{G}\right]$ lies in a closed set of dimension two, then the resulting decomposition space is homeomorphic to $E^{3}$ provided it is a 3-manifold. These results, and others given in $\$ 7$, give partial solutions to Bing's question stated in the first paragraph of this section.

The results of this paper may be regarded as results concerning cellular maps from a 3-manifold onto a 3-manifold. If $M$ is a 3-manifold, a cellular map $f$ from $M$ into a space is a continuous function with domain $M$ such that if $y \in f[M], f^{-1}[y]$ is a cellular subset of $M$. The following is a corollary of the main result: If $f$ is a cellular map from $S^{3}$ onto a 3-manifold $N$ and the set of all singular points of $f$ is properly contained in $N$, then $N$ is homeomorphic to $S^{3}$. Other results of this type are given in $\S 8$.

Presented to the Society, November 27, 1965; received by the editors May 20, 1965 and, in revised form, May 8, 1967.

(1) Supported in part by Air Force Office of Scientific Research Grant AF-AFOSR-359-63 and National Science Foundation Grant No. GP-3857. 
Some previous results concerning point-like decompositions of $E^{3}$ and $S^{3}$ for which the decomposition space is a 3-manifold are given in [1], [2], [3], [8] and[12]. Theorem 3 of [2] and the result announced in [3] are corollaries of the theorem of this paper.

The proof of the main result is given in $\S 6$. In $\S \S 3,4$ and 5 , some preliminary lemmas are established. Sections 7 and 8 give various corollaries of the main result. Those of $\S 7$ are formulated in terms of cellular decompositions while those of $\$ 8$ are stated in terms of cellular maps of compact 3-manifolds.

2. Notation and terminology. Suppose that $n$ is a positive integer. The statement that $X$ is an $n$-manifold means that $X$ is a separable metric space, each point of which has an open neighborhood homeomorphic to $E^{n}$. The statement that $X$ is an n-manifold-with-boundary means that $X$ is a separable metric space such that each point of $X$ has a neighborhood which is an $n$-cell. If $X$ is an $n$-manifold-withboundary and $p \in X$, then $p$ is a boundary point of $X$ if and only if $p$ has no open neighborhood homeomorphic to $E^{n}$. The boundary, denoted by $\mathrm{Bd} X$, of the $n$-manifold-with-boundary $X$, is the set of all boundary points of $X$. The interior, denoted Int $X$, of the $n$-manifold-with-boundary $X$, is $(X-\operatorname{Bd} X)$.

If $M$ is either a manifold or a manifold-with-boundary, by a triangulation of $M$ is meant a simplicial complex $T$ such that (1) $M=\bigcup\{t: t \in T\}$ and (2) $T$ is locally finite in the sense that each point of $M$ has a neighborhood which intersects only finitely many sets of $T$.

Suppose that $X$ is a topological space and $G$ is an upper semicontinuous decomposition of $X$. Then $X / G$ denotes the associated decomposition space, and $P$ denotes the projection map from $X$ onto $X / G$. The union of all the nondegenerate elements of $G$ is denoted by $H_{G}$.

If for some positive integer $n, X$ is either $E^{n}$ or $S^{n}$, then the statement that the subcontinuum $A$ of $X$ is point-like (in $X$ ) means that if $p$ is any point of $X$, then $X-A$ is homeomorphic to $X-\{p\}$.

Suppose that $n$ is a positive integer and $M$ is an $n$-manifold. The statement that the subset $A$ of $M$ is cellular in $M$ means that there exists a sequence $C_{1}, C_{2}, C_{3}, \ldots$ of $n$-cells in $M$ such that

(1) if $i$ is any positive integer, $C_{i+1} \subset \operatorname{Int} C_{i}$, and

(2) $A=\bigcap_{i=1}^{\infty} C_{i}$.

It is clear that if $A$ is cellular in $M$, then $A$ is a compact continuum. It is known (see [15], for instance) that if $X$ is either $S^{3}$ or $E^{3}$, then a continuum $A$ of $X$ is point-like in $X$ if and only if $A$ is cellular in $X$.

If for some positive integer $n, X$ is either $E^{n}$ or $S^{n}$, the statement that $G$ is a point-like decomposition of $X$ means that $G$ is an upper semicontinuous decomposition of $X$ such that each element of $G$ is a point-like continuum in $X$. If $n$ is a positive integer and $M$ is an $n$-manifold, the statement that $G$ is a cellular decomposition of $M$ means that $G$ is an upper semicontinuous decomposition of $M$ such that each element of $G$ is cellular in $M$. 
If $D$ is a disc and $\alpha$ is an arc, the statement that $\alpha$ spans $D$ means that the endpoints of $\alpha$ belong to Bd $D$ and (Int $\alpha$ ) $\subset$ Int $D$. If $A$ is an annulus and $\alpha$ is an arc, the statement that $\alpha$ spans $A$ means that one endpoint of $\alpha$ belongs to one boundary component of $A$, the other endpoint of $\alpha$ belongs to the other boundary component of $A$, and (Int $\alpha) \subset \operatorname{Int} A$.

If $M$ is a set, $\mathrm{Cl} M$ denotes the closure of $M$.

3. Lemmas on cellular decempesitions. The lemmas of this section are of fundamental importance in the proof of the main result of this paper. Lemmas 1 and 2 are due essentially to T. M. Price [12], [13].

LEMMA 1. If $G$ is a cellular decomposition of a 3-manifold $M$ and $U$ is a simply connected open set in $M / G$, then $P^{-1}[U]$ is simply connected.

Proof. The proof given for Theorem 2.1 of [13] carries over to this case.

LEMma 2. Suppose $G$ is a cellular decomposition of a 3-manifold $M$ and $W$ is an open 3-cell in $M / G$. If $P^{-1}[W]$ lies in an open 3-cell in $M$, then $P^{-1}[W]$ is an open 3-cell.

Proof. The argument for the case $n=3$ of Theorem 2.2 of [13] carries over with no change. Since $P^{-1}[W]$ lies in an open 3-cell, it follows that $P^{-1}[W]$ is an open 3-cell.

LEMMA 3. Suppose that $G$ is a cellular decomposition of a 3-manifold $M$ such that $M / G$ is a 3-manifold $N$. Suppose that $T$ is a triangulation of $N, \sigma$ is a 3-simplex of $T$, and $P^{-1}[\sigma]$ lies in an open 3-cell $U$ in $M$. Then if $V$ is any neighborhood of $\sigma$ in $N$, there is a polyhedral 3-cell $C$ in $N$ such that $\sigma \subset \operatorname{Int} C, C \subset V, P^{-1}[C] \subset U$, and $P^{-1}$ [Int $C$ ] is an open 3-cell.

Proof. There is a polyhedral 3-cell $C$ in $N$ such that $C$ is a regular neighborhood of $\sigma$ in $N, \sigma \subset$ Int $C, C \subset V$, and $P^{-1}[C] \subset U$. By Lemma $2, P^{-1}$ [Int $C$ ] is an open 3-cell.

\section{Lemmas on polybedral 2-spheres.}

LEMMA 4. Suppose that $X$ is a polyhedral 2-sphere in a triangulated 3-manifold $M$ such that $X$ separates $M$. Suppose that $x$ and $y$ are distinct points of $X$, and $\gamma$ is $a$ polygonal simple closed curve such that $x$ and $y$ belong to $\gamma$, and distinct components of $\gamma-\{x, y\}$ lie in different components of $M-X$. Suppose that $\Delta$ is a polyhedral singular disc such that $\gamma=\mathrm{Bd} \Delta$; and $\Delta$ and $X$ are in relative general position. Then there is an arc $\alpha$ from $x$ to $y$ and contained in $\Delta \cap X$.

Proof. Let $\Delta_{0}$ be a 2-simplex and let $f$ be a continuous function from $\Delta_{0}$ onto $\Delta$ such that $f \mid \operatorname{Bd} \Delta_{0}$ is a homeomorphism from $\operatorname{Bd} \Delta_{0}$ onto $\gamma$. Let $x_{0}$ and $y_{0}$ be points of Bd $\Delta_{0}$ such that $f\left(x_{0}\right)=x$ and $f\left(y_{0}\right)=y$. Let $p$ and $q$ be points of different components of $\gamma-X$, and let $p_{0}$ and $q_{0}$ be points of $\mathrm{Bd} \Delta_{0}$ such that $f\left(p_{0}\right)=p$ and $f\left(q_{0}\right)=q$. Note that $\left\{x_{0}, y_{0}\right\}$ separates $p_{0}$ from $q_{0}$ on $\mathrm{Bd} \Delta_{0}$. 
There is a component $A$ of $f^{-1}[\Delta \cap X]$ such that $A$ separates $p_{0}$ and $q_{0}$ in $\Delta_{0}$. For suppose not. Since $f^{-1}[\Delta \cap X]$ has only finitely many components, then, if no one of them separates $p_{0}$ and $q_{0}$ in $\Delta_{0}$, then $f^{-1}[\Delta \cap X]$ does not separate $p_{0}$ and $q_{0}$ in $\Delta_{0}$ [10, Chapter IV, Theorem 19]. There is, in that case, an arc $\beta$ in $\Delta_{0}$ from $p_{0}$ to $q_{0}$ and lying in $\Delta_{0}-f^{-1}[\Delta \cap X]$. Then $f[\beta]$ is a continuum in $\Delta$ joining $p$ and $q$ and disjoint from $X$. Since $p$ and $q$ belong to different components of $M-X, f[\beta]$ intersects $X$. This is a contradiction, so there is a component $A$ of $f^{-1}[\Delta \cap X]$ such that $A$ separates $p_{0}$ and $q_{0}$ on $\Delta_{0}$. Further, both $x_{0}$ and $y_{0}$ belong to $A$ since clearly $A$ contains at least two distinct points of $\operatorname{Bd} \Delta_{0}$ and $\left\{x_{0}, y_{0}\right\}=\left(\operatorname{Bd} \Delta_{0}\right)$ $\cap f^{-1}[\Delta \cap X]$.

Hence $f[A]$ is a connected finite graph contained in $\Delta \cap X$ and containing $x$ and $y$. Then $f[A]$ contains an arc $\alpha$ from $x$ to $y$ satisfying the conclusion of Lemma 4 .

Suppose that $C$ is a polyhedral 3-cell in a triangulated 3-manifold $M$, and $\Delta$ is a polyhedral singular disc in $M$. The statement that $\Delta$ is in normal position relative to $C$ means that (1) $(\mathrm{Bd} \Delta) \cap(\mathrm{Bd} C)$ is a polygonal arc $\alpha$ and (2) $\Delta$ is the union of a polyhedral disc $E$ and a polyhedral singular disc $F$ such that (a) $\alpha \subset \mathrm{Bd} E$, (b) $E \cap C$ $=\alpha$, (c) $\mathrm{Bd} F$ misses $C$, (d) $(\mathrm{Bd} E) \cap(\mathrm{Bd} F)$ is an arc $\beta$ such that $\beta$ misses $\alpha$, and (e) $F$ and $\mathrm{Bd} C$ are in relative general position.

Lemma 5. Suppose that $C$ is a polyhedral 3-cell in a triangulated 3-manifold $M$, and $A$ is a polyhedral annulus on $\mathrm{Bd} C$. Suppose that $n>1$ and $\Delta_{1}, \Delta_{2}, \ldots$, and $\Delta_{n}$ are mutually disjoint polyhedral singular discs in $M$ such that if $i=1,2, \ldots$, or $n$, (1) $\Delta_{i}$ is in normal position relative to $C$, (2) $(\mathrm{Bd} C) \cap\left(\mathrm{Bd} \Delta_{i}\right)$ is an arc $\alpha_{i}$ spanning $A$, and (3) $\Delta_{i} \cap(\operatorname{Bd} C) \subset(\operatorname{Int} A) \cup\left(\mathrm{Bd} \alpha_{i}\right)$. Then if $U$ is any open neighborhood, in $M$, of Int $A$, there exist mutually disjoint polyhedral singular discs $\Delta_{1}^{\prime}, \Delta_{2}^{\prime}, \ldots$, and $\Delta_{n}^{\prime}$ such that if $i=1,2, \ldots$, or $n$, (1) $\mathrm{Bd} \Delta_{i}^{\prime}=\mathrm{Bd} \Delta_{i}$, (2) $\Delta_{i}^{\prime} \cap C=\alpha_{i}$, (3) $\Delta_{i}^{\prime} \subset \Delta_{i} \cup U$, and $\Delta_{i}^{\prime}$ is in normal position relative to $C$.

Proof. If $i=1,2, \ldots$, or $n$, let $E_{i}$ be a polyhedral disc and $F_{i}$ be a polyhedral singular disc such that $\Delta_{i}=E_{i} \cup F_{i}, \alpha_{i} \subset \mathrm{Bd} E_{i}$, and $E_{i}$ and $F_{i}$ satisfy conditions relative to $C$ and one another required in the definition of normal position. In particular, Bd $F_{i}$ misses $C$.

Let $\Delta_{0}$ be a 2-simplex and if $i=1,2, \ldots$, or $n$, let $f_{i}$ be a continuous function from $\Delta_{0}$ onto $F_{i}$ such that (1) $f_{i} \mid \mathrm{Bd} \Delta_{0}$ is a homeomorphism from $\mathrm{Bd} \Delta_{0}$ onto $\mathrm{Bd} F_{i}$ and (2) $f$ is locally a piecewise-linear homeomorphism. If $i=1,2, \ldots$, or $n$, each component of $f_{i}^{-1}\left[(\mathrm{Bd} C) \cap F_{i}\right]$ is a simple closed curve lying in Int $\Delta_{0}$. If $\mu$ is such a simple closed curve, $f_{i}[\mu]$ will be called a curve of intersection of $F_{i}$ with $\mathrm{Bd} C$.

Since $\Delta_{1}, \Delta_{2}, \ldots$, and $\Delta_{n}$ are mutually disjoint, it follows that if $i=1,2, \ldots$, or $n$, $F_{i} \cap(\mathrm{Bd} C)$ lies in (Int $\left.A\right)-\left(\alpha_{1} \cup \cdots \cup \alpha_{i-1} \cup \alpha_{i+1} \cup \cdots \cup \alpha_{n}\right)$. Further, each component of (Int $A)-\left(\alpha_{1} \cup \cdots \cup \alpha_{i-1} \cup \alpha_{i+1} \cup \cdots \cup \alpha_{n}\right)$ is an open disc.

Suppose that $i=1,2, \ldots$, or $n$, and $\gamma$ is a curve of intersection of $F_{i}$ with $\mathrm{Bd} C$. With the aid of [17, Chapter VI, Theorem 3.11], it may be shown that there exists a simple closed curve $J_{y}$ on Bd $C$ such that (1) $J_{y}$ lies in some component $D_{y}$ of 
(Int $A)-\left(\alpha_{1} \cup \cdots \cup \alpha_{i-1} \cup \alpha_{i+1} \cup \cdots \cup \alpha_{n}\right)$, (2) the component $C_{\gamma}$ of $F_{i} \cap \operatorname{Bd} C$ containing $\gamma$ lies in the disc $B_{\gamma}$ in $D_{\gamma}$ bounded by $J_{\gamma}$, and (3) the component of $B_{y}-C_{y}$ containing $J_{y}$ is disjoint from $\bigcup_{k=1}^{n} F_{k}$.

Since each of $F_{1}, F_{2}, \ldots$, and $F_{n}$ has only finitely many curves of intersection with $\mathrm{Bd} C$, there is a positive integer $j$ such that $j \leqq n$ and a curve of intersection $\lambda$ of $F_{j}$ with $\mathrm{Bd} C$ such that if $k \neq j, B_{\lambda}$ is disjoint from $\Delta_{k}$. Now $\lambda$ bounds a singular $\operatorname{disc} \delta_{\lambda}$ in Int $B_{\lambda}$. We cut $F_{j}$ along $\lambda$ and then replace the singular disc on $F_{j}$ bounded by $\lambda$ by $\delta_{\lambda}$. We then deform the resulting singular disc slightly to one side of $\mathrm{Bd} C$, staying in $U$ during the deformation. This is done in such a way that there results a polyhedral singular disc $F_{j}^{(1)}$ such that (1) $\mathrm{Bd} F_{j}^{(1)}=\mathrm{Bd} F_{j}$ and $F_{j}^{(1)} \subset F_{j} \cup U$, (2) $\lambda$ is not a curve of intersection of $F_{j}^{(1)}$ with $\mathrm{Bd} C$, and (3) if $\gamma$ is a curve of intersection of $F_{j}^{(1)}$ with $\mathrm{Bd} C$, then $\gamma$ is a curve of intersection of $F_{j}$ with $\mathrm{Bd} C$. Observe that if $k \neq j, F_{j}^{(1)}$ and $\Delta_{k}$ are disjoint.

The singular discs $F_{1}, \ldots, F_{j-1}, F_{j}^{(1)}, F_{j+1}, \ldots$, and $F_{n}$ satisfy the conditions assumed for $F_{1}, F_{2}, \ldots$, and $F_{n}$. Hence the process described above may be repeated. After finitely many steps, there result mutually disjoint polyhedral singular $\operatorname{discs} F_{1}^{\prime}, F_{2}^{\prime}, \ldots$, and $F_{n}^{\prime}$ such that if $i=1,2, \ldots$, or $n$, (1) $\operatorname{Bd} F_{1}^{\prime}=\operatorname{Bd} F_{i}$, (2) $F_{i}^{\prime} \subset F_{i} \cup U$, and (3) $F_{i}^{\prime}$ misses $C$. If $i=1,2, \ldots$, or $n$, adjust $F_{i}^{\prime}$ slightly so that if $\Delta_{i}^{\prime}$ is the union of $E_{i}$ and the adjusted $F_{i}^{\prime}, \Delta_{i}^{\prime}$ is a singular disc in normal position relative to $C$. Then $\Delta_{1}^{\prime}, \Delta_{2}^{\prime}, \ldots$, and $\Delta_{n}^{\prime}$ satisfy the conclusion of Lemma 5 .

5. Constructing homeomorphisms. In order to prove the theorem, we shall need to construct a homeomorphism from one 3-manifold $M$ onto another 3-manifold $N$. In this section, we shall show that such a homeomorphism can be constructed provided we can embed into $M$ the carrier of the 2-skeleton of some triangulation of $N$ so that certain conditions are satisfied. These conditions are stated in Lemma 8. Lemmas 6 and 7 are preliminary lemmas for Lemma 8.

Once Lemma 8 is established, the proof of the theorem is reduced to the construction of a suitable embedding into $M$ of the carrier of the 2-skeleton of some triangulation of $N$.

Lemma 6. Suppose that $M$ is a 3-manifold and $T$ is a triangulation of $M$. If $T_{2}$ is the carrier of the 2-skeleton of $T$ and $\sigma$ is any 3-simplex of $T$, then $T_{2}-(\mathrm{Bd} \sigma)$ is connected.

Proof. Suppose that $v$ and $v^{\prime}$ are any two distinct vertices of $T$ not on $\sigma$. It will be shown that $T_{2}-\mathrm{Bd} \sigma$ contains a connected set containing both $v$ and $v^{\prime}$.

Since $M$ is connected, there is a chain $\left\{s_{1}, s_{2}, \ldots, s_{m}\right\}$ of 1 -simplexes of $T$ such that

(1) $v$ is a vertex of $s_{1}$ and $v^{\prime}$ is a vertex of $s_{m}$,

(2) $s_{1} \cup s_{2} \cup \cdots \cup s_{m}$ is an $\operatorname{arc} A$, and

(3) $A \cap(\mathrm{Bd} \sigma)$ is either (a) a vertex $p$ of $T$ or (b) a 1-simplex $s_{i}$ of $T$.

Suppose 3(a) holds and consider the closed star St $(p)$ of $p$ in $T$. By Theorem 1 of [9], Bd $\mathrm{St}(p)$ is a 2-sphere $S$. Clearly $(\operatorname{Bd} \sigma) \cap S$ is a 2-simplex $D$. Now $A \cap S$ is 
disjoint from $D$ and it is clear that both components of $A-\{p\}$ intersect $S$. Therefore $(A-\{p\}) \cup(S-D)$ is a connected subset of $T_{2}-(\mathrm{Bd} \sigma)$ containing both $v$ and $v^{\prime}$.

Suppose 3(b) holds, and that $s_{i}=\left\langle p_{1} p_{2}\right\rangle$ where $p_{1}$ precedes $p_{2}$ on $A$ in the order from $v$ to $v^{\prime}$ on $A$. Let $A_{1}$ and $A_{2}$ be the components of $A-\left\langle p_{1} p_{2}\right\rangle$ containing $v$ and $v^{\prime}$, respectively. Let $S_{1}$ and $S_{2}$ denote $\mathrm{Bd} \mathrm{St}\left(p_{1}\right)$ and $\mathrm{Bd} \operatorname{St}\left(p_{2}\right)$, respectively; $S_{1}$ and $S_{2}$ are 2-spheres.

There is a 3-simplex $\sigma^{\prime}$ of $T$ distinct from $\sigma$ and having both $p_{1}$ and $p_{2}$ as vertices. Let $q$ be a vertex of $\sigma^{\prime}$ not on $\sigma ; q$ is distinct from both $p_{1}$ and $p_{2}$. Clearly $q \in S_{1} \cap S_{2}$. If $j=1$ or $2,(\mathrm{Bd} \sigma) \cap S_{i}$ is a disc $D_{i}$ and $q \notin D_{i}$. Further, if $j=1$ or $2, A_{j}$ intersects $S_{j}-D_{j}$. Hence

$$
A_{1} \cup A_{2} \cup\left(S_{1}-D_{1}\right) \cup\left(S_{2}-D_{2}\right)
$$

is a connected subset of $T_{2}-(\mathrm{Bd} \sigma)$ containing both $v$ and $v^{\prime}$.

Hence for each two vertices $v$ and $v^{\prime}$ of $T$ not on $\sigma$, there is a connected subset of $T_{2}-(\mathrm{Bd} \sigma)$ containing both $v$ and $v^{\prime}$. Since each component of $T_{2}-(\mathrm{Bd} \sigma)$ contains at least one vertex of $T$, it follows that $T_{2}-(\operatorname{Bd} \sigma)$ is connected.

LEMMA 7. If $M$ and $N$ are 3-manifolds, $\Sigma$ and $T$ are triangulations of $M$ and $N$, respectively, and $\Sigma$ and $T$ are isomorphic (as complexes), then $M$ and $N$ are homeomorphic.

Proof. Let $\varphi$ be an isomorphism from $\Sigma$ onto $T$. Define a function $f$ as follows: If $v$ is a vertex of $\Sigma$, then $f(v)=\varphi(v)$. Extend $f$ homeomorphically to the 1-simplexes of $\Sigma$, then to the 2-simplexes of $\Sigma$, and finally to the 3-simplexes of $\Sigma$ so that if $\sigma$ is any simplex of $\Sigma, f[\sigma]=\varphi(\sigma)$. It is clear that the function $f$ so constructed is from $M$ onto $N$ and is one-to-one. Since $\Sigma$ is locally finite, $f$ is continuous, and since $T$ is locally finite, $f^{-1}$ is continuous. Hence $f$ is a homeomorphism from $M$ onto $N$.

LemMA 8. Suppose that $M$ and $N$ are triangulated connected 3-manifolds, $T$ is a triangulation of $N$, and $T_{2}$ is the carrier of the 2-skeleton of $T$. Suppose there exist a piece-wise linear homeomorphism $h$ from $T_{2}$ into $M$ and a locally finite collection $\mathscr{U}$ of open 3-cells in $M$ such that (1) if $\sigma$ is any 3-simplex of $T$, there is an open 3-cell $U_{\sigma}$ of $\mathscr{U}$ such that $h[\mathrm{Bd} \sigma] \subset U_{\sigma}$, but $h\left[T_{2}\right]$ does not lie in $U_{\sigma}$, and (2) if $\sigma$ and $\tau$ are distinct 3-simplexes of $T, U_{\sigma}^{*} \neq U_{\tau}$. Then $M$ and $N$ are homeomorphic.

Proof. We shall construct a triangulation $\Sigma$ of $M$ such that $\Sigma$ and $T$ are isomorphic. Lemma 8 will then follow from Lemma 7.

For each 3-simplex $\sigma$ of $T$, let $S_{\sigma}$ denote $h[\mathrm{Bd} \sigma]$. By hypothesis, there is an open 3-cell $U_{\sigma}$ of $\mathscr{U}$ such that $S_{\sigma} \subset U$. Let $I_{\sigma}$ be the component of $U_{\sigma}-S_{\sigma}$ whose closure relative to $U_{\sigma}$ is compact. Then $S_{\sigma} \cup I_{\sigma}$ is a polyhedral 3-cell in $M$. It is easy to see that $S_{\sigma}$ separates $I_{\sigma}$ and $M-\left(S_{\sigma} \cup I_{\sigma}\right)$, and that $M-\left(S_{\sigma} \cup I_{\sigma}\right)$ is connected.

We shall show now that $I_{\sigma}$ and $h\left[T_{2}\right]$ are disjoint. By Lemma $6, T_{2}-\mathrm{Bd} \sigma$ is connected. Hence $h\left[T_{2}\right]-S_{\sigma}$ is connected. If $T_{2}$ intersects $I_{\sigma}$, then $h\left[T_{2}\right]-S_{\sigma}$ lies in $I_{\sigma}$ and hence in $U_{\sigma}$. It would follow then that $h\left[T_{2}\right] \subset U_{\sigma}$, but this is contrary to hypothesis. 
Next we shall show that if $\sigma$ and $\tau$ are distinct 3-simplexes of $T, I_{\sigma}$ and $I_{\tau}$ are disjoint. Suppose $I_{\sigma}$ and $I_{\tau}$ intersect. Since $I_{\sigma}$ and $I_{\tau}$ are connected and, as shown above, neither intersects the boundary of the other, one lies in the other. Suppose that $I_{\sigma} \subset I_{\tau}$. Since $S_{\sigma} \neq S_{\tau}$, then $I_{\sigma} \neq I_{\tau}$. Hence some boundary point of $I_{\sigma}$ lies in $I_{\tau}$. This is contradictory since $h\left[T_{2}\right]$ and $I_{\tau}$ are disjoint. Similarly, it is impossible that $I_{\tau} \subset I_{\sigma}$. Hence $I_{\sigma}$ and $I_{\tau}$ are disjoint.

Let $\Sigma$ denote $\{h[t]: t$ is a 0 -, 1-, or 2-simplex of $T\} \cup\left\{S_{\sigma} \cup I_{\sigma}: \sigma\right.$ is a 3-simplex of $T$ \}. It is clear that $\Sigma$ and $T$ are isomorphic as complexes. We shall show that $\Sigma$ is a triangulation of $M$.

First, $\Sigma$ is a locally finite collection. This follows from the following facts: (1) $\mathscr{U}$ is locally finite. (2) If $\sigma$ and $\tau$ are distinct 3-simplexes of $T, U_{\sigma} \neq U_{\tau}$. (3) For each 3-simplex $\sigma$ of $T, S_{\sigma} \cup I_{\sigma} \subset U_{\sigma}$.

Now let $\Sigma_{3}$ denote $\bigcup\left\{S_{\sigma} \cup I_{\sigma}: \sigma\right.$ is a 3-simplex of $\left.T\right\}$. It will be shown that $\Sigma_{3}=M$. Suppose that $\Sigma_{3}$ is a proper subset of $M$. By Lemma 7 , there is a homeomorphism $g$ from $N$ onto $\Sigma_{3}$. Since $\Sigma$ is a locally finite collection, $\Sigma_{3}$ is closed in $M$. Since $M$ is connected, there is a point $x$ of $\Sigma_{3}$ which is a limit point of $M-\Sigma_{3}$. There is an open 3-cell $V$ in $N$ such that $V$ is an open neighborhood in $N$ of $g^{-1}(x)$. Since $M$ is a 3-manifold, $g[V]$ is open in $M$ and hence intersects $M-\Sigma_{3}$. But $g[V] \subset \Sigma_{3}$, and this is a contradiction. Therefore $\Sigma_{3}=M$.

It then follows that $\Sigma$ is a triangulation of $M$. Since $\Sigma$ and $T$ are isomorphic, then by Lemma 7, $M$ and $N$ are homeomorphic.

\section{Proof of the main result.}

THEOREM. Suppose that $M$ is a connected 3-manifold, $G$ is a cellular decomposition of $M$ such that $M / G$ is a 3-manifold $N$, and $N$ has a triangulation $T$ such that (1) no vertex of $T$ belongs to the closure of $P\left[H_{G}\right]$ and (2) if $\sigma$ is any 3-simplex of $T$, there is an open 3-cell in $M$ containing $P^{-1}[\sigma]$. Then $M$ and $N$ are homeomorphic.

The proof of the theorem is based on Lemma 8. $M$ has a triangulation [5], [9]. We shall construct (1) a piece-wise linear embedding $h$ of the carrier $T_{2}$ of the 2skeleton of $T$ into $M$ and (2) a locally finite collection $\mathscr{U}$ of open 3-cells in $M$ such that $h$ and $\mathscr{U}$ satisfy the hypothesis of Lemma 8 . It will then follow that $M$ and $N$ are homeomorphic.

In order to construct the embedding $h$ of $T_{2}$ into $M$, we construct a polyhedral 2-complex $\Sigma$ in $M$ such that $\Sigma$ and the 2-skeleton of $T$ are isomorphic complexes and $\Sigma$ satisfies certain additional technical conditions.

Speaking roughly, we shall construct the 2-complex $\Sigma$ by using $P^{-1}$ to "lift" $T_{2}$ into $M$. It should be emphasized, however, that $P^{-1}$ is by no means necessarily a local homeomorphism. Hence our procedure will be, roughly, to use $P^{-1}\left[T_{2}\right]$ as a guide in constructing $\Sigma$. For each simplex $s$ of $T$ of dimension 0,1 , or 2 , we approximate $P^{-1}[s]$ with a polyhedral set. These approximations may have singularities. From these polyhedral sets we will then construct the 2-complex $\Sigma$. 
The major difficulty is in constructing the 2-simplexes of $\Sigma$ and insuring that they fit together properly. As an aid toward the construction of $\Sigma$, we divide $T_{2}$ into two pieces and construct the 2-simplexes of $\Sigma$ in two corresponding pieces. We construct a tubular neighborhood $K$ of the carrier $T_{1}$ of the 1-skeleton of $T$. We then use $P^{-1}$ to "lift" $K$ into $M$ to yield a tubular neighborhood $K^{\prime}$ of the carrier $\Sigma_{1}$ of the 1skeleton of $\Sigma$. We construct each 2-simplex of $\Sigma$ in two steps: the part of the 2-simplex in $K^{\prime}$, and the part not in $K^{\prime}$.

Therefore the proof can be divided into three main parts. The first is the construction of the tubular neighborhood $K$ of $T_{1}$ and certain associated sets. The second is the construction of the tubular neighborhood $K^{\prime}$ of $\Sigma_{1}$ in $M$. The last is the construction of the simplexes of $\Sigma$ and of the embedding $h$ of $T_{2}$ into $M$.

Proof of the theorem. $N$ has, by hypothesis, a triangulation $T$, and the statement that a subset of $N$ is polygonal or polyhedral means with respect to $T$. Later in the proof we shall choose a triangulation of $M$.

In the proof of the theorem, we use a process of thickening arcs, discs, and other subsets of $N$. This process, not described in detail in this paper, may be carried out with the use of Whitehead's theory of regular neighborhoods and suitable subdivisions of the triangulation $T$.

The 2-complex to be constructed in $M$ is denoted by $\Sigma$. If $i=0,1$, or $2, T_{i}$ and $\Sigma_{i}$ denote the carriers of the $i$-skeletons of $T$ and $\Sigma$, respectively.

Since the proof of the theorem is long, we have broken the proof into 12 steps.

Step 1. For each 3-simplex $\sigma$ of $T$, we shall construct an open 3-cell $U_{\sigma}$ in $M$ having certain properties. First we choose certain 3-cells in $N$. If $\sigma$ is a 3-simplex of $T$, there is, by hypothesis, an open 3-cell $U_{\sigma}^{0}$ in $M$ such that $P^{-1}[\sigma] \subset U_{\sigma}^{0}$. Since $G$ is upper semicontinuous, there is an open set $V_{\sigma}^{0}$ in $N$ containing $\sigma$ and such that $P^{-1}\left[V_{\sigma}^{0}\right] \subset U_{\sigma}^{0}$. Let $\mathscr{W}$ be the set of all 3-simplexes of $T ; \mathscr{W}$ is a countable locally finite collection of closed sets covering $N$. It may be shown that there is a locally finite collection $\mathscr{A}$ of open sets such that if $\sigma \in \mathscr{W}$, there is a set of $\mathscr{A}$ containing $\sigma$ and contained in $V_{\sigma}^{0}$. For each 3-simplex $\sigma$ of $T$, let $W_{\sigma}^{0}$ be an open set of $\mathscr{A}$ such that $\sigma \subset W_{\sigma}^{0}$ and $W_{\sigma}^{0} \subset V_{\sigma}^{0}$.

If $\sigma$ is a 3-simplex of $T$, there is, by Lemma 3, a 3-cell $X_{\sigma}$ in $N$ such that $\sigma \subset$ Int $X_{\sigma}$, $P^{-1}$ [Int $\left.X_{\sigma}\right] \subset U_{\sigma}^{0}, P^{-1}$ [Int $X_{\sigma}$ ] is an open 3-cell, and $X_{\sigma}$ lies in the open star (in $T$ ) of $\sigma$. For each 3-simplex $\sigma$ of $T$, let $U_{\sigma}$ denote $P^{-1}$ [Int $X_{\sigma}$ ]. Then

$$
\left\{U_{\sigma}: \sigma \text { is a 3-simplex of } T\right\}
$$

is a locally finite collection of open 3-cells covering $M$ such that if $\sigma$ is any 3-simplex of $T, P^{-1}[\sigma] \subset U_{\sigma}$. Note that for each 3-simplex $\sigma$ of $T, X_{\sigma}$ contains no vertex of $T$ distinct from the vertices of $\sigma$.

Step 2. We shall construct a tubular neighborhood $K$ of $T_{1}$. Let $v_{1}, v_{2}, \ldots$ denote the distinct vertices of $T$. Let $C_{1}, C_{2}, \ldots$ be mutually disjoint polyhedral 3-cells in $N$ such that if $v_{i}$ is any vertex of $T$, then

(1) $v_{i} \in \operatorname{Int} C_{i}$ and $C_{i}$ is disjoint from the closure of $P\left[H_{G}\right]$, 


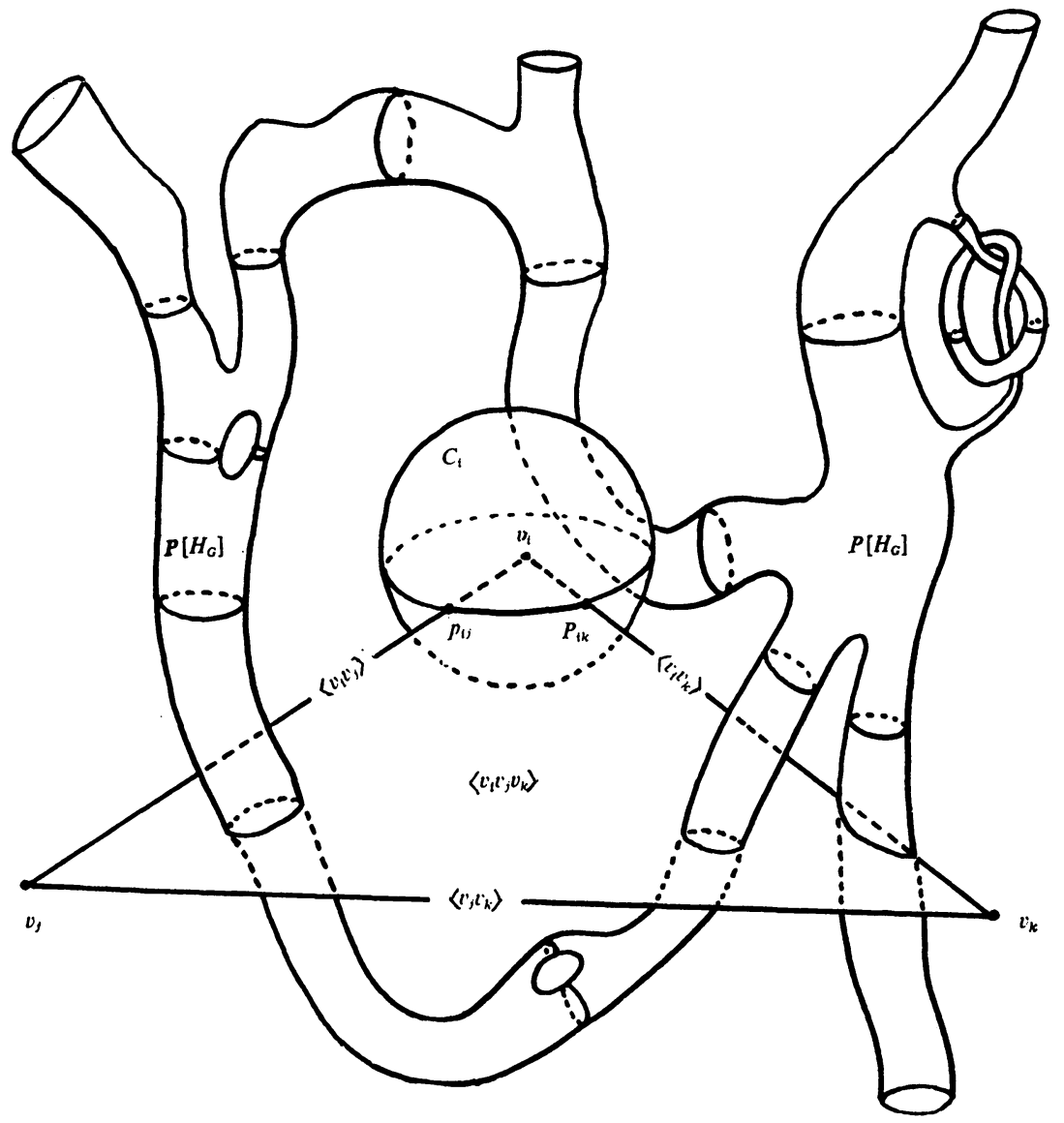

FIGURE 1

(2) If $v_{j}$ is a vertex of $T$ such that $\left\langle v_{i} v_{j}\right\rangle$ is a 1 -simplex of $T$, there is a point $p_{i j}$ of Int $\left\langle v_{i} v_{j}\right\rangle$ such that $\left\langle v_{i} v_{j}\right\rangle \cap \mathrm{Bd} C=\left\{p_{i j}\right\}$,

(3) $C_{i}$ intersects various 1- and 2-simplexes of $T$ as indicated in Figure 1, and

(4) if $\sigma$ is any 3-simplex of $T$ having $v_{i}$ as a vertex, then $C_{i} \subset$ Int $X_{\sigma}$, but if $\sigma$ is any 3-simplex of $T$ not having $v_{i}$ as a vertex, then $C_{i}$ and $X_{\sigma}$ are disjoint.

Let $\left\langle v_{i_{1}} v_{j_{1}}\right\rangle,\left\langle v_{i_{2}} v_{j_{2}}\right\rangle, \ldots$ denote the distinct 1 -simplexes of $T$. Let $L_{i_{1} j_{1}}, L_{i_{2} j_{2}}, \ldots$ be mutually disjoint polyhedral 3 -cells in $N$ such that if $\left\langle v_{i} v_{j}\right\rangle$ is a 1-simplex of $T$,

(1) $L_{i j}=L_{j i}$,

(2) $C_{i} \cap L_{i j}$ is a polyhedral disc $\Delta_{i j}$ such that $\Delta_{i j}=\operatorname{Bd} C_{i} \cap \operatorname{Bd} L_{i j}$ and $p_{i j}$ $\in$ Int $\Delta_{i j}$

(3) if $t=1,2, \ldots$ and $t$ is neither $i$ nor $j$, then $L_{i j}$ and $C_{t}$ are disjoint,

(4) $L_{i j}$ intersects $\left\langle v_{i} v_{j}\right\rangle$ and various 2-simplexes of $T$ as indicated in Figures 2 and 3 , and

(5) if $\sigma$ is any 3-simplex of $T$ having $\left\langle v_{i} v_{j}\right\rangle$ as an edge, then $L_{i j} \subset$ Int $X_{\sigma}$. 


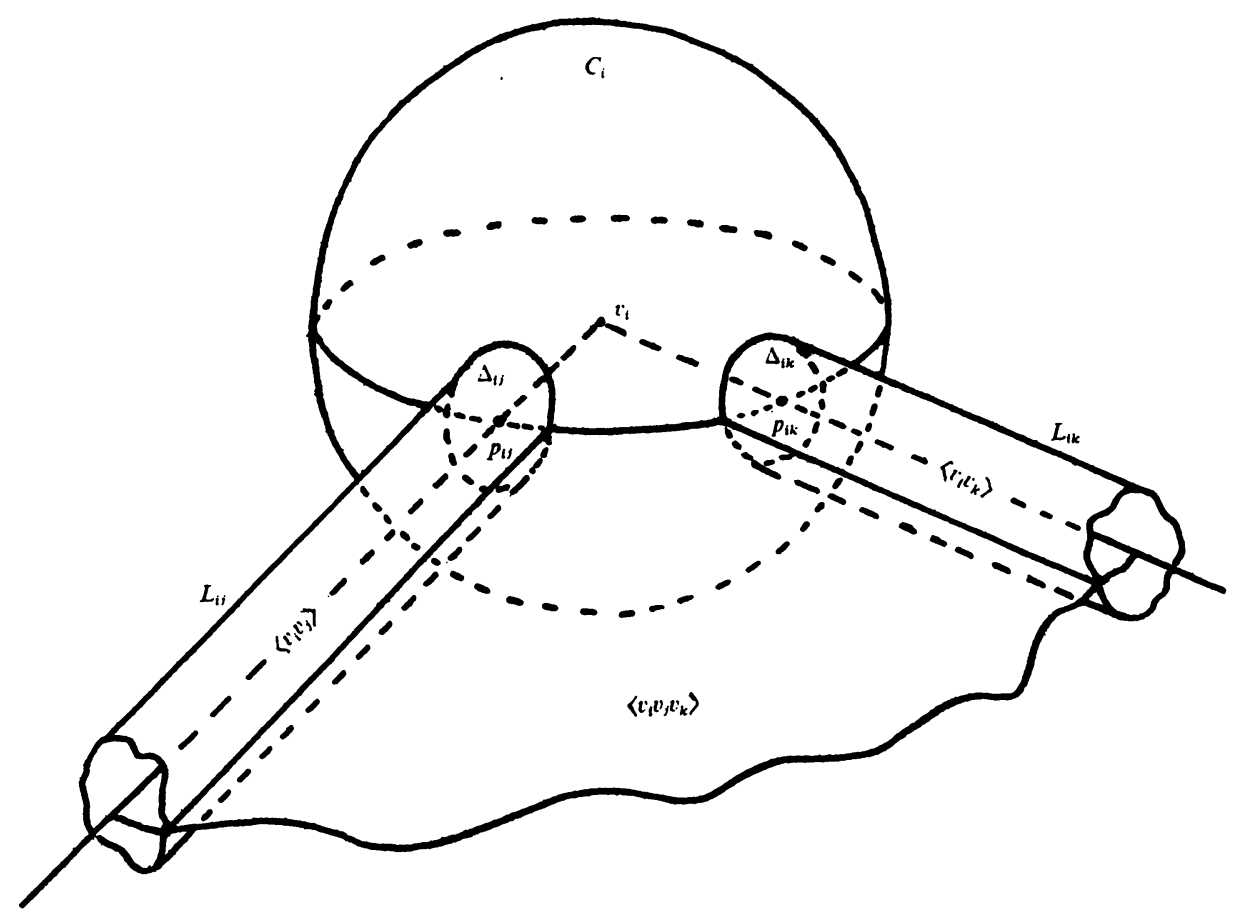

FIGURE 2

The tubular neighborhood $K$ of $T_{1}$ is

$$
\left(\bigcup\left\{C_{i}: v_{i} \text { is a vertex of } T\right) \cup\left(\bigcup\left\{L_{i j}:\left\langle v_{i} v_{j}\right\rangle \text { is a 1-simplex of } T\right\}\right) .\right.
$$

Step 3. Suppose $\left\langle v_{i} v_{j}\right\rangle$ is a 1-simplex of $T$. We shall describe some subsets of Bd $L_{i j}$. Let $A_{i j}$ denote

$$
\text { Bd } L_{i j}-\left(\text { Int } \Delta_{i j} \cup \operatorname{Int} \Delta_{j i}\right)
$$

$A_{i j}$ is an annulus whose boundary curves are $\operatorname{Bd} \Delta_{i j}$ and $\operatorname{Bd} \Delta_{j i}$. Note that $A_{i j}=A_{j i}$. If $\left\langle v_{i} v_{j} v_{k}\right\rangle$ is any 2-simplex having $\left\langle v_{i} v_{j}\right\rangle$ as an edge, let $\pi_{i j k}$ denote the arc $A_{i j}$ $\cap\left\langle v_{i} v_{j} v_{k}\right\rangle ; \pi_{i j k}=\pi_{j i k}$ and $\pi_{i j k}$ spans $A_{i j}$. See Figure 4.

Recall that $C_{i}$ and $C_{j}$ are disjoint from $\mathrm{Cl} P\left[H_{G}\right]$. There exist, then, disjoint annuli $B_{i j}$ and $B_{f t}$ on $A_{i j}$ such that

(1) $B_{i j}$ and $B_{j i}$ are disjoint from $\mathrm{Cl} P\left[H_{G}\right]$,

(2) $\mathrm{Bd} \Delta_{i j}$ is one boundary component of $B_{i j}$, and $\mathrm{Bd} \Delta_{j i}$ is one boundary component of $B_{f i}$, and

(3) $B_{i j}$ and $B_{i j}$ intersect the various arcs $\pi_{i j k}$ as indicated in Figure 4.

Let $F_{i j}$ denote $\mathrm{Cl}\left[A_{i j}-\left(B_{i j} \cup B_{j i}\right)\right] ; F_{i j}$ is an annulus contained in Int $A_{i j}$.

Let $\left\langle v_{i} v_{j} v_{k_{1}}\right\rangle,\left\langle v_{i} v_{j} v_{k_{2}}\right\rangle, \ldots$, and $\left\langle v_{i} v_{j} v_{k_{d(1)}}\right\rangle$ denote the 2-simplexes of $T$ having $\left\langle v_{i} v_{j}\right\rangle$ as an edge. There exist disjoint polyhedral discs $Q_{i j k_{1}}, Q_{i j k_{2}}, \ldots$, and $Q_{i j k_{a(i j)}}$ such that if $k=k_{1}, k_{2}, \ldots$, or $k_{\mathrm{d}(t)}$, 


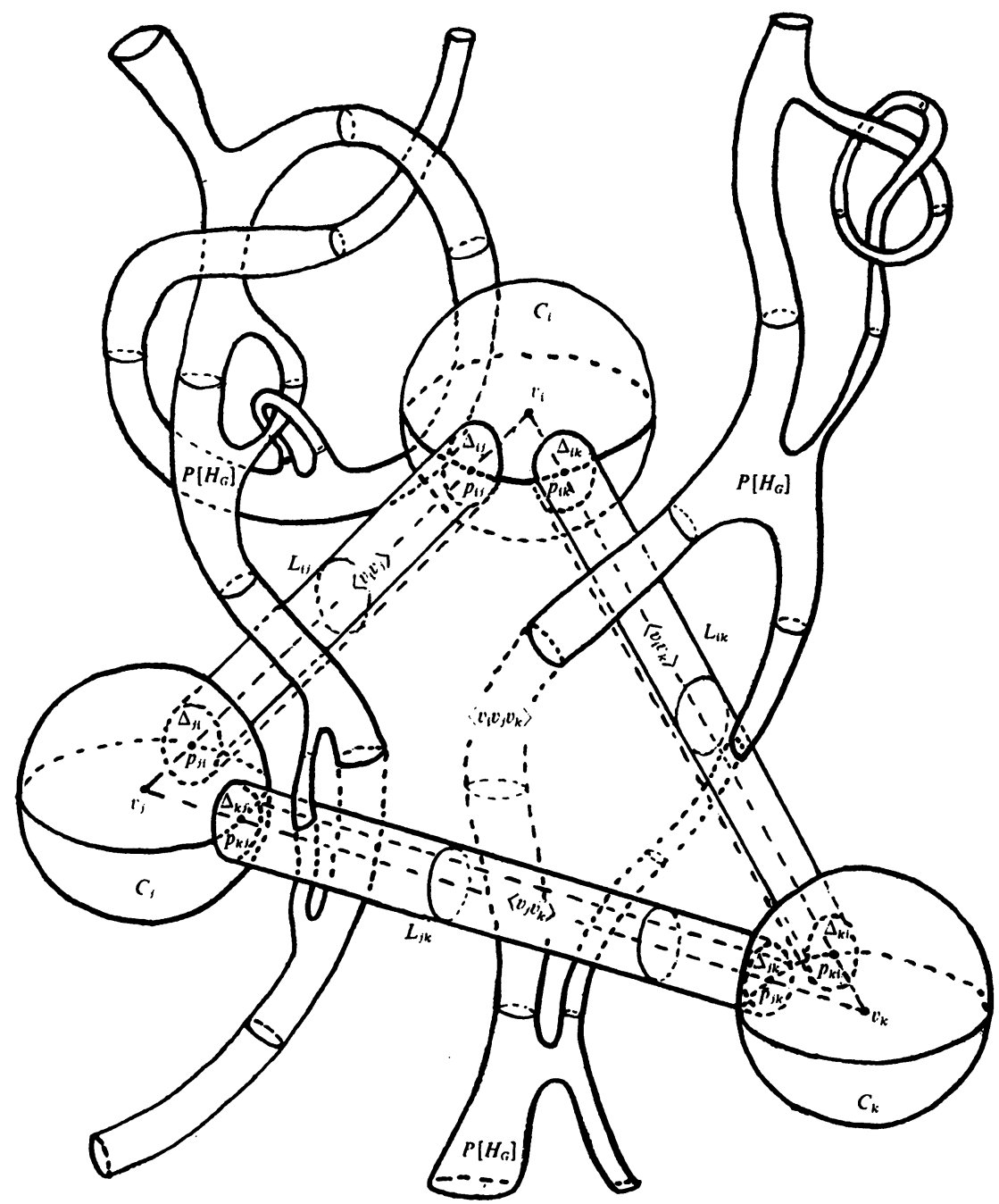

Figure 3

(1) $Q_{i j k} \subset A_{i j}$ and $\mathrm{Bd} Q_{i j k}$ is the union of two spanning arcs of $A_{i j}$, an arc on $\operatorname{Bd} \Delta_{i j}$, and an arc on $\operatorname{Bd} \Delta_{j i}$,

(2) the arc $\pi_{i j k}$ spans $Q_{i j k}$, and

(3) $Q_{i j k}$ lies on $A_{i j}$ as shown in Figure 5.

Let $\mathscr{E}_{i j}$ denote the set of all $E$ such that either

(1) for some positive integer $k$ such that $\left\langle v_{i} v_{j} v_{k}\right\rangle$ is a 2-simplex of $T, E=F_{i j} \cap$ $Q_{i j k}$, or

(2) $E$ is the closure of a component of

$$
F_{i j}-\bigcup\left\{Q_{i j k}:\left\langle v_{i} v_{j} v_{k}\right\rangle \text { is a 2-simplex of } T\right\} .
$$

Each set of $\mathscr{E}_{i j}$ is a disc lying on $F_{i j}$ and such that $E \cap \operatorname{Bd} F_{i j}$ is the union of two 


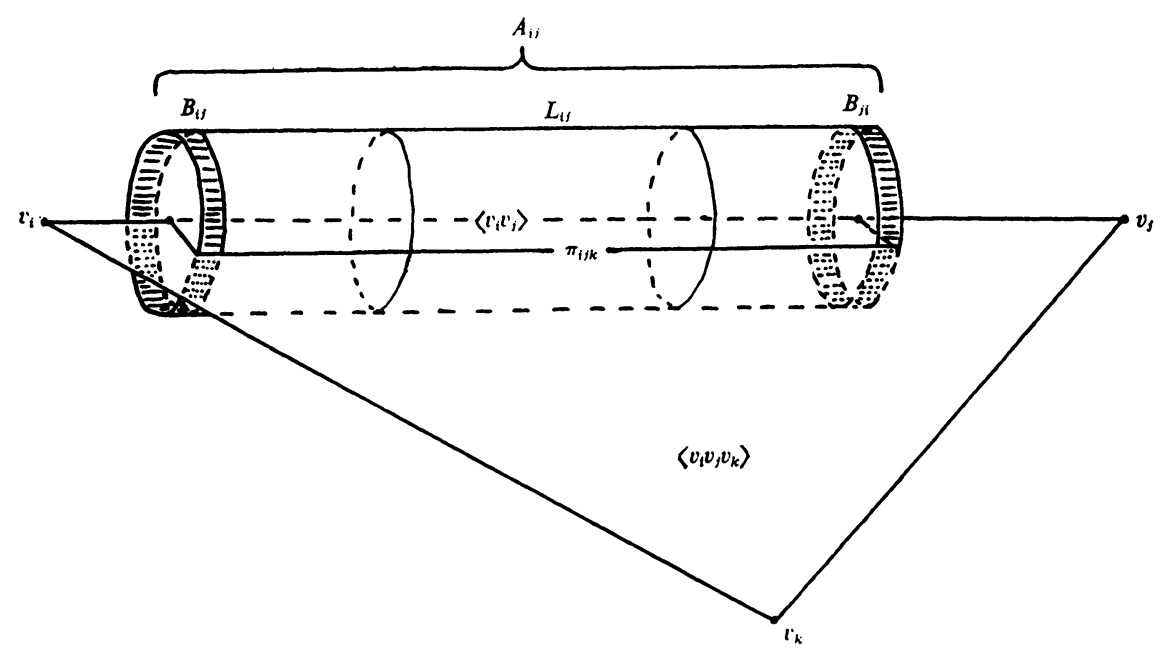

FIGURE 4

disjoint arcs, one on each boundary component of $F_{i j}$. Further, there is a cyclic ordering

$$
E_{i j 1}, E_{i j 2}, \ldots, E_{i j \lambda_{i j}}
$$

of the sets of $\mathscr{E}_{i j}$ such that if each of $k$ and $l$ is a positive integer not greater than $\lambda_{i j}$, then

(1) $E_{i j k}$ and $E_{i j l}$ intersect if and only if either $|k-l| \leqq 1$ or $\{k, l\}=\left\{1, \lambda_{i j}\right\}$, and

(2) if $E_{i j k}$ and $E_{i j l}$ intersect, then $E_{i j k} \cap E_{i j l}$ is an arc spanning the annulus $F_{i j}$.

Step 4. We shall now describe certain open subsets of $N$ associated with Bd $K$. Consider the annuli $F_{i_{1} j_{1}}, F_{i_{2} j_{2}}, \ldots$ There exist mutually disjoint open sets (in $N$ ) $F_{i_{1} j_{1}}^{*}, F_{i_{2} j_{2}}^{*}, \ldots$ such that if $t=1,2, \ldots$,

(1) $F_{i_{t} j_{t}} \subset F_{i_{t} j_{t}}^{*}$

(2) if $v_{k}$ is a vertex of $T, F_{t_{t} j_{t}}^{*}$ and $C_{k}$ are disjoint,

(3) if $s \neq t, L_{i_{s} j_{s}}$ and $F_{i_{t} j_{t}}^{*}$ are disjoint,

(4) $F_{t_{t} j_{t}}^{*}$ is disjoint from $T_{1}$, and

(5) $F_{i_{t} j_{t}}^{*}$ is disjoint from each 2-simplex of $T$ not having $\left\langle v_{i_{t}} v_{j_{t}}\right\rangle$ as an edge.

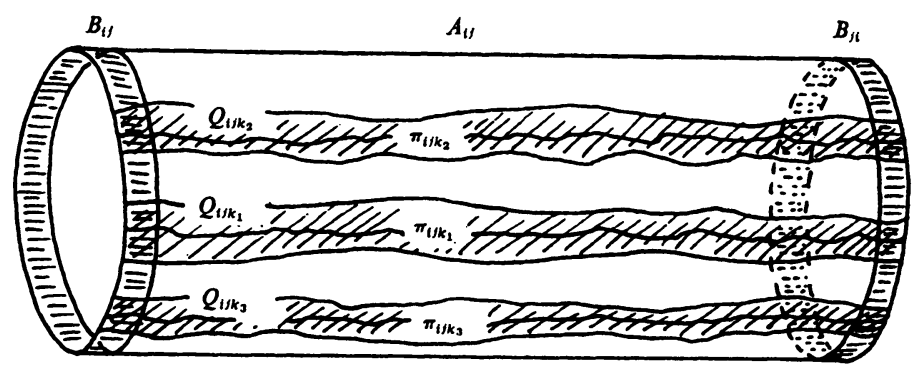

Figure 5 
Suppose now that $\left\langle v_{i} v_{j}\right\rangle$ is a 1 -simplex of $T$. There exist connected open sets $W_{i j 1}, W_{i j 2}, \ldots$, and $W_{i j \lambda_{i j}}$ such that

(1) if $t=1,2, \ldots$, or $\lambda_{i j}$,

(i) $W_{i j t}$ is obtained by a slight thickening of $E_{i j t}$,

(ii) $E_{i j t} \subset \bar{W}_{i j t}$, and $W_{i j t} \subset F_{i j}^{*}$,

(iii) $W_{i j t}$ is simply connected, and

(iv) if $\sigma$ is any 3-simplex of $T$ having $\left\langle v_{i} v_{j}\right\rangle$ as an edge, then $W_{i j t} \subset$ Int $X_{\sigma}$, and (2) if $s=1,2, \ldots$, or $\lambda_{i j}$ and $t=1,2, \ldots$, or $\lambda_{i j}$, then $W_{i j s}$ and $W_{i j t}$ intersect if and only if $E_{i j s}$ and $E_{i j t}$ intersect, and if $W_{i j s}$ and $W_{i j t}$ intersect, their common part is connected.

Let $W_{i j}$ denote $\bigcup_{t=1}^{\lambda t_{1}} W_{i j t}$. Then $W_{i j}$ satisfies the following conditions:

(1) $F_{i j} \subset W_{i j}$.

(2) $W_{i j}$ intersects no 2-simplex of $T$ not having $\left\langle v_{i} v_{j}\right\rangle$ as an edge.

(3) For each vertex $v_{k}$ of $T, C_{k}$ and $W_{i j}$ are disjoint.

(4) If $\left\langle v_{s} v_{t}\right\rangle$ is a 1-simplex of $T$ distinct from $\left\langle v_{i} v_{j}\right\rangle, L_{s t}$ and $W_{i j}$ are disjoint.

(5) If $\sigma$ is any 3-simplex of $T$ having $\left\langle v_{i} v_{j}\right\rangle$ as an edge, then $W_{i j} \subset$ Int $X_{\sigma}$.

(6) There is a polyhedral 3-cell $Y_{i j}$ contained in $L_{i j}-W_{i j}$ and such that

(i) $Y_{i j} \cap \Delta_{i j}$ and $Y_{i j} \cap \Delta_{j i}$ are discs such that $p_{i j}$ and $p_{j i}$ belong to their respective interiors, and

(ii) $Y_{i j}$ meets each 2-simplex of $T$ having $\left\langle v_{i} v_{j}\right\rangle$ as an edge as indicated in Figure 6.

Before we start on Step 5, we introduce some notation to be used throughout the remainder of the proof. If $X$ is any subset of $N$, then $\tilde{X}$ denotes $P^{-1}[X]$. If $p$ is any point of $N$ and there is only one point $x$ of $M$ such that $P(x)=p$, then $\tilde{p}$ denotes $x$.

Step 5. We first choose a triangulation of $M$. We may, by [5], [9], regard $M$ as a triangulated manifold. It is easy to see that for each vertex $v_{i}, \mathrm{Bd} \tilde{C}_{i}$ is locally tame [7], and for each 1-simplex $\left\langle v_{i} v_{j}\right\rangle, \widetilde{B}_{i j}$ and $\widetilde{B}_{j i}$ are locally tame. By [7, Theorem 8], there is a triangulation of $M$ relative to which the $\widetilde{C}_{i}$ 's, the $\widetilde{B}_{i}$ 's, and various associated sets, are polyhedral. Choose one such triangulation of $M$; the statement that a subset of $M$ is polygonal or polyhedral means relative to this particular triangulation of $M$.

Now for each 1-simplex $\left\langle v_{i} v_{j}\right\rangle$ of $T$, we shall construct an annulus $A_{i j}^{\prime}$ in $M$ that corresponds to the annulus $A_{i j}$ in $N$.

Suppose that $\left\langle v_{i} v_{j}\right\rangle$ is a 1-simplex of $T$. Let $x_{1}, x_{2}, \ldots$, and $x_{\lambda_{j}}$ be points of $B_{i j} \cap F_{i j}$, and let $y_{1}, y_{2}, \ldots$, and $y_{\lambda_{i j}}$ be points of $B_{j i} \cap F_{i j}$ such that if $t=1,2, \ldots$, or $\lambda_{i j}-1$, the arc $E_{i j t} \cap E_{i j(t+1)}$ has endpoints $x_{t}$ and $y_{t}$, and the arc $E_{i j 1} \cap E_{i j \lambda_{i j}}$, has endpoints $x_{\lambda_{t}}$ and $y_{\lambda_{i j}}$.

Consider the sets $\tilde{W}_{i j 1}, \tilde{W}_{i j 2}, \ldots$, and $\tilde{W}_{i j \lambda_{i j}}$. If $t=1,2, \ldots$, or $\lambda_{i j}, W_{i j t} \cap W_{i j(t+1)}$ is connected and hence so is $\tilde{W}_{i j t} \cap \tilde{W}_{i j(t+1)}$. There is, therefore, a polygonal arc $\mu_{i j t}^{0}$ from $\tilde{x}_{t}$ to $\tilde{y}_{t}$, lying in $\tilde{W}_{i j t} \cap \tilde{W}_{i j(t+1)}$. Similarly, there is a polygonal arc $\mu_{i j \lambda_{i j}}^{0}$ from $\tilde{x}_{\lambda_{t j}}$ to $\tilde{y}_{\lambda_{t j}}$ lying in $\tilde{W}_{i j 1} \cap \tilde{W}_{i j \lambda_{i j}}$. 


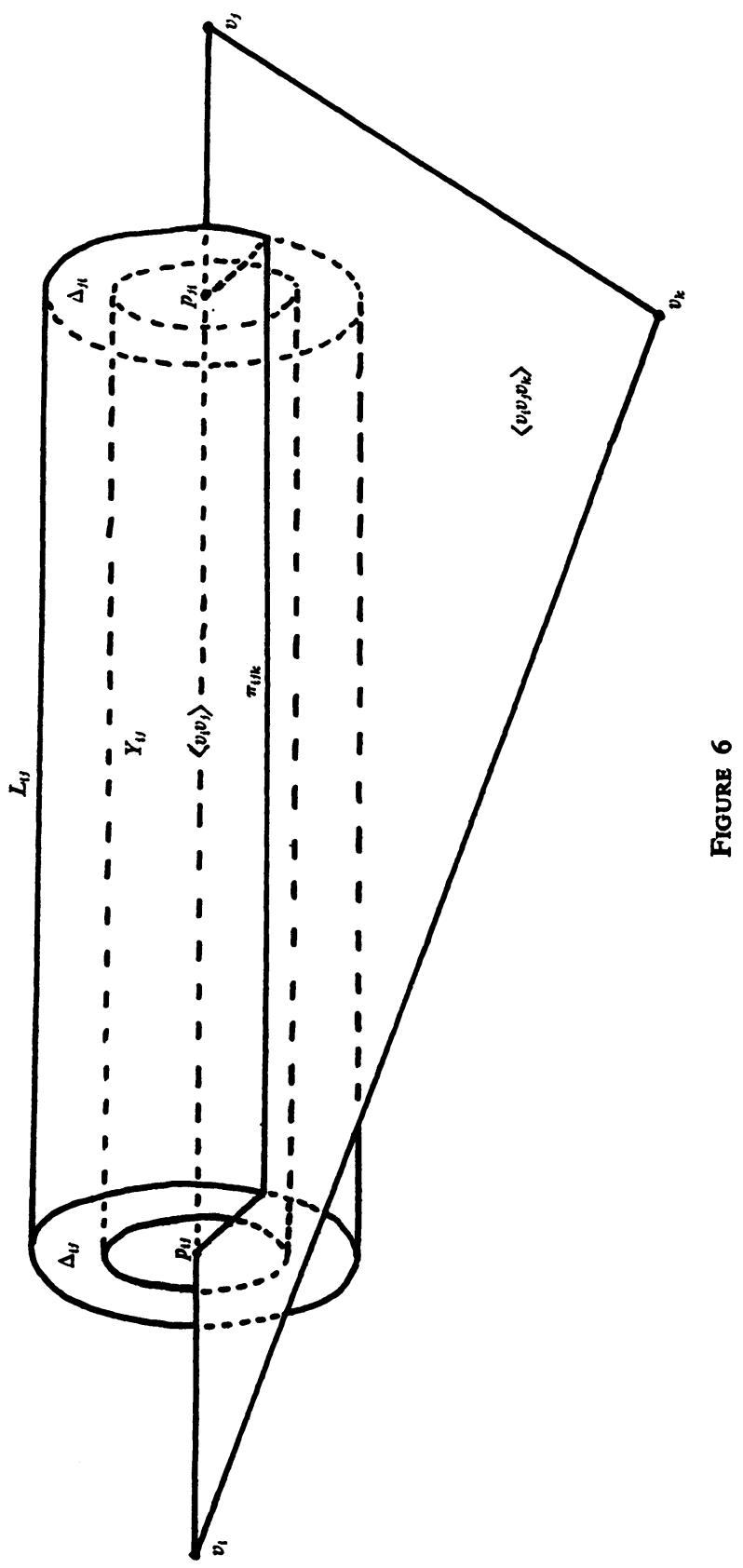


If $t=1,2, \ldots$, or $\lambda_{i j}-1$, let $\tilde{x}_{t} \tilde{x}_{t+1}$ and $\tilde{y}_{t} \tilde{y}_{t+1}$ denote arcs of $\tilde{B}_{i j} \cap \tilde{F}_{i j}$ and $\widetilde{B}_{j i}$ $\cap \tilde{F}_{i j}$, respectively, lying in $\tilde{W}_{i j t}$, and let $\gamma_{i j t}^{0}$ denote

$$
\tilde{x}_{t} \tilde{x}_{t+1} \cup \mu_{i j t}^{0} \cup \mu_{i j(t+1)}^{0} \cup \tilde{y}_{t} \tilde{y}_{t+1} \text {. }
$$

Let $\tilde{x}_{1} \tilde{x}_{\lambda_{i j}}$ and $\tilde{y}_{1} \tilde{y}_{\lambda_{i j}}$ denote arcs of $\tilde{B}_{i j} \cap \tilde{F}_{i j}$ and $\widetilde{B}_{j i} \cap \tilde{F}_{i j}$, respectively, lying in $\tilde{W}_{i j \lambda_{i j}}$, and let $\gamma_{i j \lambda_{i j}}^{0}$ denote

$$
\tilde{x}_{1} \tilde{x}_{\lambda_{i j}} \cup \mu_{i j 1}^{0} \cup \mu_{i j \lambda_{i j}}^{0} \cup \tilde{y}_{1} \tilde{y}_{\lambda_{i j}} \cdot
$$

If $t=1,2, \ldots$, or $\lambda_{i j}$, then $\gamma_{i j t}^{0} \subset \tilde{W}_{i j t}$; since $W_{i j t}$ is simply connected, then by Lemma 1 , so is $\tilde{W}_{i j t}$. Therefore $\gamma_{i j t}^{0}$ bounds a polyhedral singular disc $\Gamma_{i j t}$ contained in $\tilde{W}_{i j t}$.

Let $\Omega_{i j}$ denote

$$
\widetilde{B}_{i j} \cup \widetilde{B}_{j i} \cup\left(\bigcup_{t=1}^{\lambda i j} \Gamma_{i j}\right)
$$

Then $\Omega_{i j}$ is a polyhedral singular annulus such that

(1) $\operatorname{Bd} \Omega_{i j}=\left(\operatorname{Bd} \tilde{\Delta}_{i j}\right) \cup\left(\operatorname{Bd} \tilde{\Delta}_{j i}\right)$,

(2) Bd $\Omega_{i j}$ has a neighborhood on $\Omega_{i j}$ which contains no point of singularity of $\Omega_{i j}$, and

(3) $\Omega_{i j} \subset \tilde{W}_{i j} \cup \widetilde{B}_{i j} \cup \widetilde{B}_{j i}$.

Let $Z_{i j}$ denote Int $\left(C_{i} \cup C_{j} \cup Y_{i j}\right)$. Then $Z_{i j}$ is a connected open set, $v_{i}$ and $v_{j}$ belong to $Z_{i j}$, and $Z_{i j}$ and $W_{i j} \cup B_{i j} \cup B_{j i}$ are disjoint.

It is clear that $\tilde{Z}_{i j}$ contains a polygonal arc $\varphi_{i j}$ from $\tilde{v}_{i}$ to $\tilde{v}_{j}$ such that $\varphi_{i j}$ contains exactly one point of each of Int $\tilde{\Delta}_{i j}$ and Int $\tilde{\Delta}_{j i}$.

Suppose now that $k$ is some positive integer such that $\left\langle v_{i} v_{j} v_{k}\right\rangle$ is a 2-simplex of $T$. Let $\varphi_{i j k}$ denote $\varphi_{i j} \cup \varphi_{i k} \cup \varphi_{j k} ; \varphi_{i j k}$ is a polygonal closed curve. Since $\varphi_{i j k}$ contains exactly one point of each of Int $\tilde{\Delta}_{i j}$ and Int $\tilde{\Delta}_{j i}$, then $\varphi_{i j k}$ links each of Bd $\tilde{\Delta}_{i j}$ and $\operatorname{Bd} \tilde{\Delta}_{j i}$. For a definition of linking of polygonal closed curves as it is used here, and proofs of some elementary properties, see [6, pp. 480-482].

By [14], there exists a nonvoid subset $\mathscr{C}$ of $\left\{\operatorname{Bd} \tilde{\Delta}_{i}\right.$, Bd $\left.\tilde{\Delta}_{j i}\right\}$ and a nonsingular disc or annulus $A_{i j}^{\prime}$ such that

(1) the curves of $\mathscr{C}$ are the boundary curves of $A_{i j}^{\prime}$, and

(2) $A_{i j}^{\prime} \subset \tilde{W}_{i j} \cup \tilde{B}_{i j} \cup \tilde{B}_{j i}$.

Now it will be shown that $A_{i j}^{\prime}$ is not a disc. Suppose that $A_{i j}^{\prime}$ is a disc. Then Bd $A_{i j}^{\prime}$ is either Bd $\tilde{\Delta}_{i j}$ or Bd $\tilde{\Delta}_{j i}$. Suppose Bd $A_{i j}=\operatorname{Bd} \tilde{\Delta}_{i j}$. Since $\varphi_{i j k}$ links Bd $\tilde{\Delta}_{i j}$, it follows that $\varphi_{i j k}$ intersects $A_{i j}^{\prime}$. However, since $A_{i j}^{\prime} \subset \tilde{W}_{i j} \cup \widetilde{B}_{i j} \cup \widetilde{B}_{i j}, \varphi_{i j k} \subset \tilde{Z}_{i j}$ $\cup \tilde{Z}_{i k} \cup \tilde{Z}_{j k}$, and $\tilde{W}_{i j} \cup \tilde{B}_{i j} \cup \tilde{B}_{j i}$ and $\tilde{Z}_{i j} \cup \tilde{Z}_{i k} \cup \tilde{Z}_{j k}$ are disjoint, this is a contradiction. Similarly, it is impossible that $\mathrm{Bd} A_{i j}^{\prime}=\mathrm{Bd} \tilde{\Delta}_{j i}$. Therefore, $A_{i j}^{\prime}$ is an annulus, and

It is clear that

$$
\operatorname{Bd} A_{i j}^{\prime}=\operatorname{Bd} \tilde{\Delta}_{i j} \cup \operatorname{Bd} \tilde{\Delta}_{j i} \text {. }
$$

(1) $A_{i j}^{\prime} \cap \tilde{C}_{i}=$ Bd $\tilde{\Delta}_{i j}$,

(2) $A_{i j}^{\prime} \cap \widetilde{C}_{j}=\mathrm{Bd} \tilde{\Delta}_{j i}$, and 


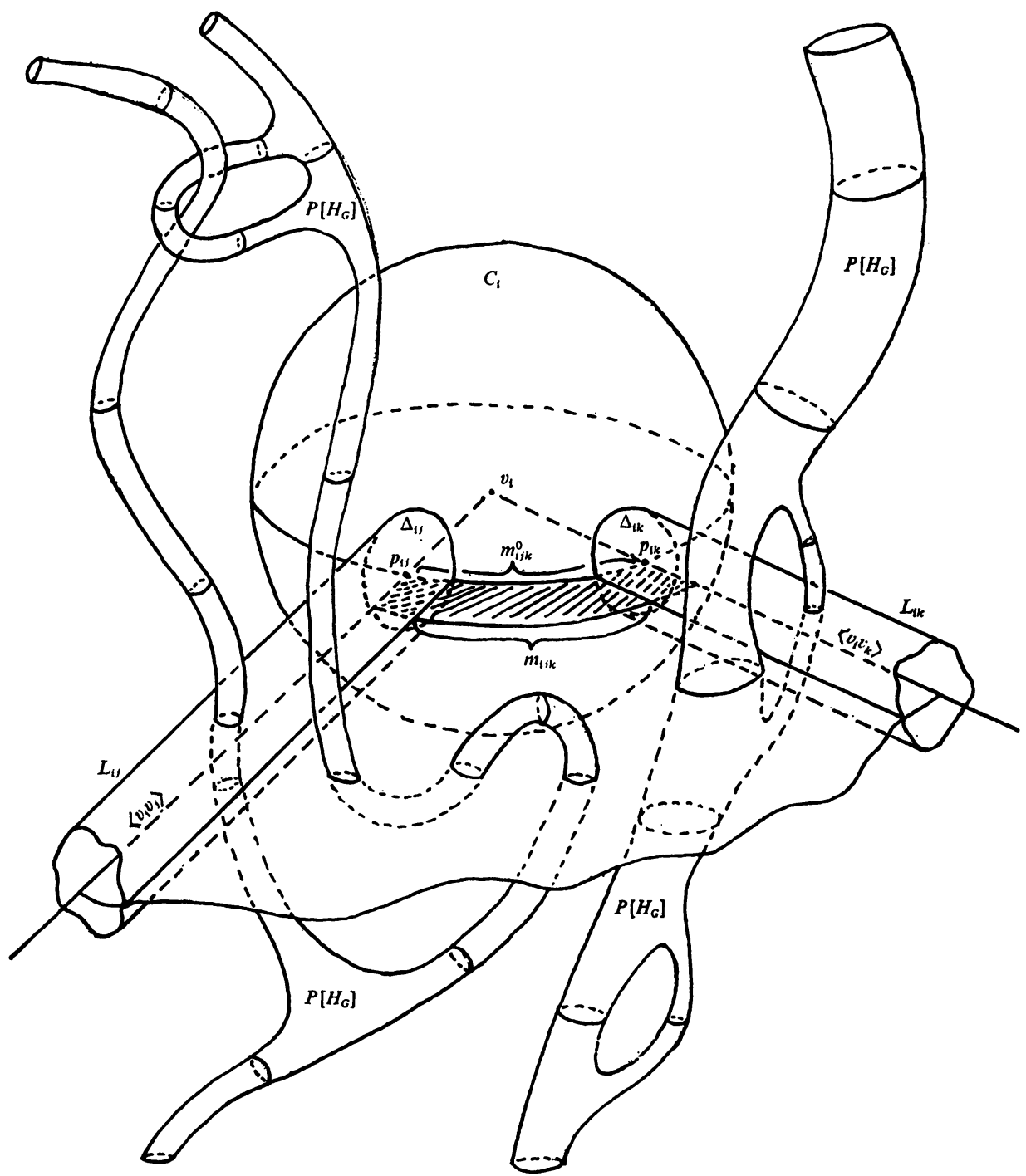

FIGURE 7

(3) if $t=1,2, \ldots$ and $t$ is neither $i$ nor $j$, then $\tilde{C}_{t}$ and $A_{i j}^{\prime}$ are disjoint.

Step 6. We may now construct a set $K^{\prime}$ in $M$ which will be a tubular neighborhood of the carrier $\Sigma_{1}$ of the 1-skeleton of $\Sigma$, and which corresponds to the tubular neighborhood $K$ in $N$.

Suppose that $\left\langle v_{i} v_{j}\right\rangle$ is a 1-simplex of $T$. It is clear that $A_{i j}^{\prime} \cup \tilde{\Delta}_{i j} \cup \tilde{\Delta}_{j i}$ is a polyhedral 2-sphere; let $S_{i j}$ denote $A_{i j}^{\prime} \cup \tilde{\Delta}_{i j} \cup \tilde{\Delta}_{j i}$. Further if $\sigma$ is any 3-simplex of $T$ such that $\left\langle v_{i} v_{j}\right\rangle$ is an edge of $\sigma$, then $S_{i j} \subset U_{\sigma}$. Hence $S_{i j}$ is the boundary of a polyhedral 3-cell $L_{i j}^{\prime}$ in $M$ such that the following conditions hold:

(1) If $\left\langle v_{s} v_{t}\right\rangle$ is a 1-simplex of $T$ distinct from $\left\langle v_{t} v_{j}\right\rangle$ then $L_{i j}^{\prime}$ and $L_{s t}^{\prime}$ are disjoint. 
(2) If $t=1,2, \ldots$, then $\widetilde{C}_{t}$ and $L_{i j}^{\prime}$ intersect if and only if $t=i$ or $t=j$, and if $t=i$ or $t=j$, then $L_{i j}^{\prime} \cap \tilde{C}_{t}$ is a disc common to $\mathrm{Bd} L_{i j}^{\prime}$ and $\mathrm{Bd} \tilde{C}_{t}$.

Let $K^{\prime}$ denote

$\left(\bigcup\left\{\tilde{C}_{i}: v_{i}\right.\right.$ is a vertex of $\left.\left.T\right\}\right) \cup\left(\bigcup\left\{L_{i j}^{\prime}:\left\langle v_{i} v_{j}\right\rangle\right.\right.$ is a 1-simplex of $\left.\left.T\right\}\right)$.

Step 7. In this step, we prepare for the construction of $\Sigma_{1}$ and $\Sigma_{2}$.

Suppose that $\left\langle v_{i} v_{j} v_{k}\right\rangle$ is a 2-simplex of $T$. Then $\left\langle v_{i} v_{j} v_{k}\right\rangle-\operatorname{Int}\left(C_{i} \cup C_{j} \cup C_{\dot{k}}\right)$ is a $\operatorname{disc} \varepsilon_{i j k}$. There is a polygonal arc $\alpha_{i j k}$ such that

(1) $\alpha_{i j k}$ spans $\varepsilon_{i j k}$, has one endpoint on $\left\langle v_{i} v_{j}\right\rangle$, the other endpoint on $\left\langle v_{i} v_{k}\right\rangle$, and is disjoint from the arc $\varepsilon_{i j k} \cap \mathrm{Bd} C_{i}$,

(2) $\alpha_{i j k}$ intersects each of $\mathrm{Bd} Y_{i j}, \mathrm{Bd} Y_{i k}, \mathrm{Bd} L_{i j}$, and $\mathrm{Bd} L_{i k}$ in one point, and $\alpha_{i j k}$ intersects $\mathrm{Bd} L_{i j}$ in one point which lies in $B_{i j}-W_{i j}$.

(3) If $m_{i j k}^{0}$ denotes the closure of the component of $\varepsilon_{i j k}-\alpha_{i j k}$ containing $\varepsilon_{i j k}$ $\cap \mathrm{Bd} C_{i}$, then $m_{i j k}^{0}$ is disjoint from the closure of $P\left[H_{G}\right]$. See Figure 7.

There are discs $m_{j i k}^{0}$ and $m_{k i j}^{0}$ satisfying analogous conditions and in addition, $m_{i j k}^{0}, m_{j i k}^{0}$, and $m_{k i j}^{0}$ are to be mutually disjoint.

Let $m_{i j k}, m_{j i k}$, and $m_{k i j}$ denote the discs $m_{i j k}^{0}$-Int $K, m_{j i k}^{0}-$ Int $K$, and $m_{k i j}^{0}$ - Int $K$, respectively. See Figure 8 .

Let $\tau_{i j}$ be the arc in Int $\left\langle v_{i} v_{j} v_{k}\right\rangle \cap \mathrm{Bd} Y_{i j}$ having one endpoint on $\alpha_{i j k}$ and the other on $\alpha_{j i k}$. Let $\tau_{i k}$ and $\tau_{j k}$ be arcs satisfying analogous conditions. Let $\alpha_{i j k}^{0}$ be the subarc of $\alpha_{i j k}$ whose endpoints are those of $\tau_{i j}$ and $\tau_{i k}$ on $\alpha_{i j k}$. Let $\theta_{i j k}$ denote $\tau_{i j} \cup \tau_{i k} \cup \tau_{j k} \cup \alpha_{i j k}^{0} \cup \alpha_{j i k}^{0} \cup \alpha_{k i j}^{0} . \theta_{i j k}$ is a polygonal simple closed curve lying in Int $\left\langle v_{i} v_{j} v_{k}\right\rangle$. Let $\delta_{i j k}$ be the subdisc of $\left\langle v_{i} v_{j} v_{k}\right\rangle$ bounded by $\theta_{i j k}$. Observe that

(1) $\delta_{i j k} \subset$ Int $\left\langle v_{i} v_{j} v_{k}\right\rangle$ and

(2) $\delta_{i j k}$ is disjoint from each of $C_{i}, C_{j}$, and $C_{k}$.

Suppose that $\left\langle v_{i_{1}} v_{j_{1}} v_{k_{1}}\right\rangle,\left\langle v_{i_{2}} v_{j_{2}} v_{k_{2}}\right\rangle, \ldots$ are the distinct 2-simplexes of $T$. There exist mutually disjoint open sets $V_{i_{1} j_{1} k_{1}}, V_{i_{2} j_{2} k_{2}}, \ldots$ such that if $\left\langle v_{i} v_{j} v_{k}\right\rangle$ is a 2 simplex of $T$, then

(1) $V_{i j k}$ is obtained by a slight thickening of $\delta_{i j k}, \delta_{i j k} \subset V_{i j k}$, and $V_{i j k}$ is simply connected,

(2) if $v_{t}$ is a vertex of $T, C_{t}$ and $V_{i j k}$ are disjoint,

(3) $V_{i j k} \cap T_{2} \subset \operatorname{Int}\left\langle v_{i} v_{j} v_{k}\right\rangle$,

(4) $V_{i j k} \cup$ Int $Y_{i j} \cup$ Int $Y_{i k} \cup$ Int $Y_{j k}$ is a simply connected, connected open set,

(5) each of $V_{i j k} \cap$ Int $Y_{i j}, V_{i j k} \cap$ Int $Y_{i k}$, and $V_{i j k} \cap$ Int $Y_{j k}$ is connected,

(6) each of $V_{i j k} \cap m_{i j k}, V_{i j k} \cap m_{j i k}$, and $V_{i j k} \cap m_{k i j}$ is connected, and

(7) if $\sigma$ is any 3-simplex having $\left\langle v_{i} v_{j} v_{k}\right\rangle$ as a face, then $V_{i j k} \subset$ Int $X_{\sigma}$. See Figure 9; this shows a part of $V_{i j k}$ near $\mathrm{Bd} C_{i}$. Bd $C_{i}$ is omitted and parts of $A_{i j}$ and $A_{i k}$ are cut away.

Step 8. We shall now construct, for each 1-simplex $\left\langle v_{i} v_{j}\right\rangle$ of $T$, $\operatorname{arcs} \pi_{i j k}^{\prime}$ on $A_{i j}^{\prime}$ that correspond to the arcs $\pi_{i j k}$ of $A_{i j}$.

Suppose that $\left\langle v_{i} v_{j}\right\rangle$ is a 1-simplex of $T$ and that $\left\langle v_{i} v_{j} v_{k}\right\rangle$ is a 2-simplex of $T$ having 


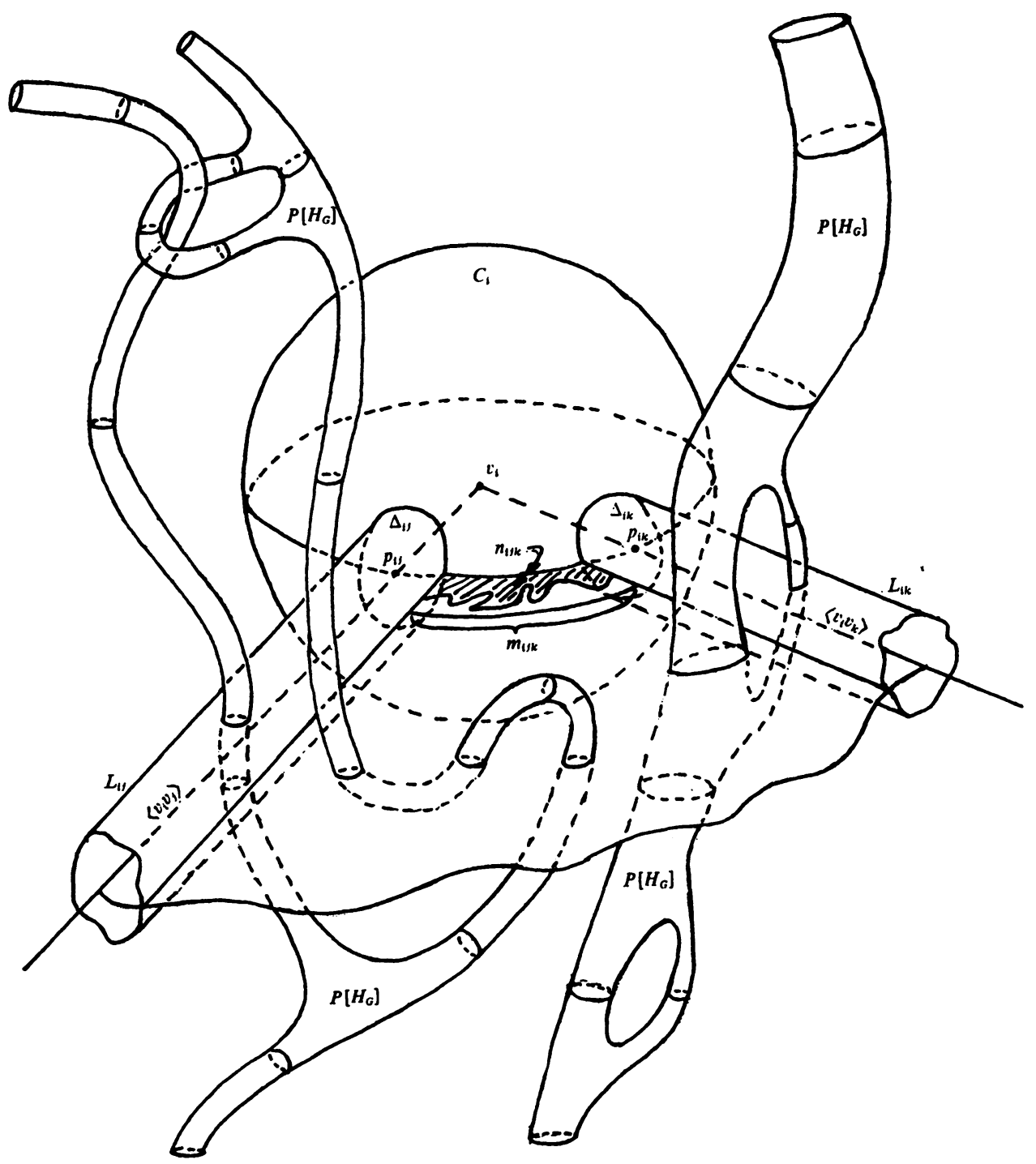

FIGURE 8

$\left\langle v_{i} v_{j}\right\rangle$ as an edge. Let $w_{i j}$ and $w_{i k}$ be the endpoints of the arc $\alpha_{i j k}$ on $\left\langle v_{i} v_{j}\right\rangle$ and $\left\langle v_{i} v_{k}\right\rangle$, respectively. Let $w_{j i}$ and $w_{j k}$ be the endpoints of the arc $\alpha_{j i k}$ on $\left\langle v_{j} v_{i}\right\rangle$ and $\left\langle v_{j} v_{k}\right\rangle$, respectively. Let $w_{k i}$ and $w_{k j}$ be the endpoints of the arc $\alpha_{k i j}$ on $\left\langle v_{k} v_{i}\right\rangle$ and $\left\langle v_{k} v_{j}\right\rangle$, respectively.

Let $\rho_{i j k}$ be a simple closed curve in $M$ which is a union of arcs as follows:

(1) An arc in $P^{-1}$ [Int $Y_{i j}$ ] from $\tilde{w}_{i j}$ to $\tilde{w}_{j i}$.

(2) An arc in $P^{-1}$ [Int $Y_{i k}$ ] from $\tilde{w}_{i k}$ to $\tilde{w}_{k i}$.

(3) An arc in $P^{-1}$ [Int $Y_{j k}$ ] from $\tilde{w}_{j k}$ to $\tilde{w}_{k j}$.

(4) The $\operatorname{arcs} \tilde{\alpha}_{i j k}, \tilde{\alpha}_{j i k}$, and $\tilde{\alpha}_{k i j}$. 


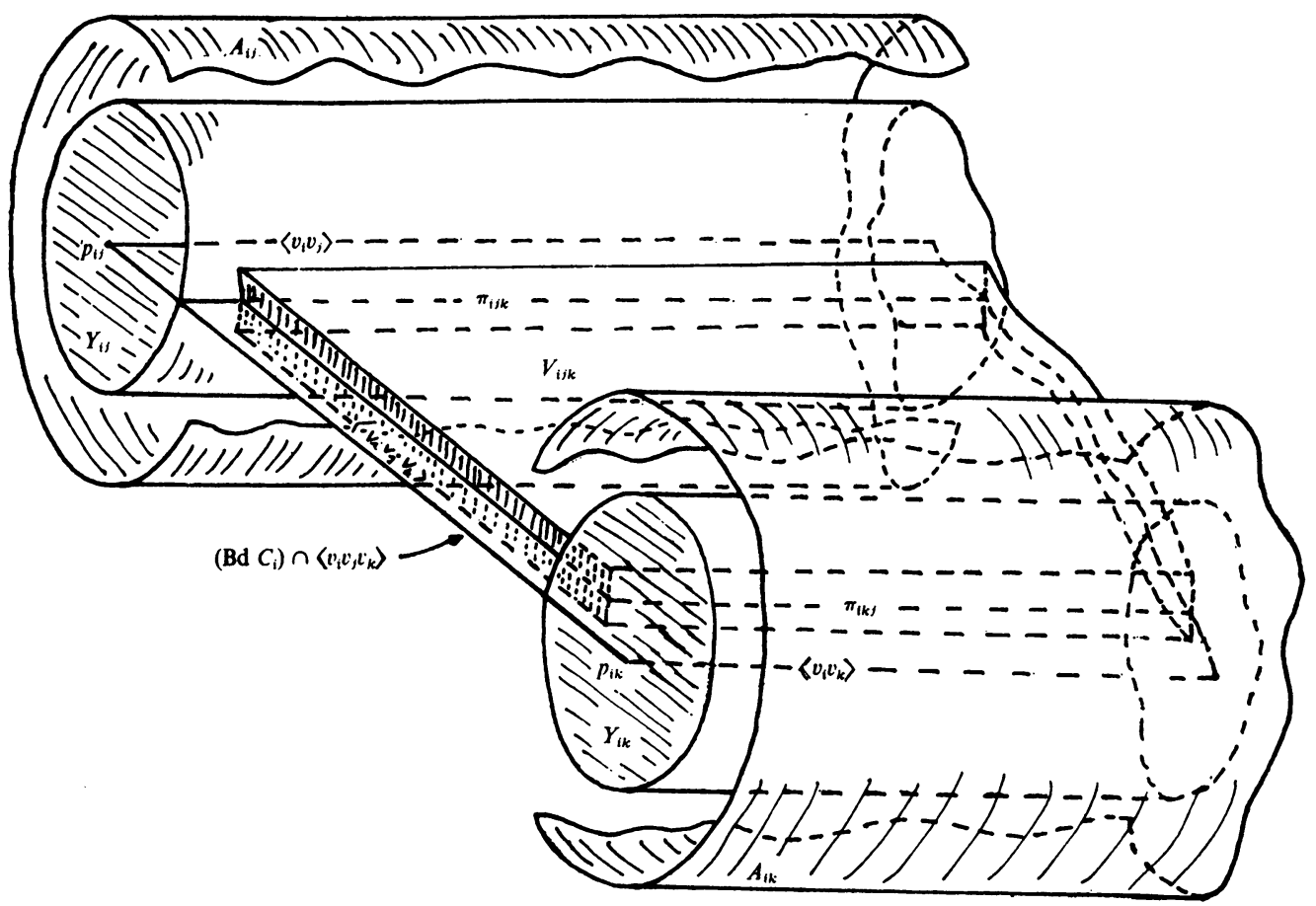

FIGURE 9

The simple closed curve $\rho_{i j k}$ lies in

$$
P^{-1}\left[V_{i j k} \cup \operatorname{Int} Y_{i j} \cup \operatorname{Int} Y_{i k} \cup \operatorname{Int} Y_{j k}\right] \text {. }
$$

Now consider the arc $\alpha_{i j k}$ and Bd $L_{i j}$. It follows from the construction of $\alpha_{i j k}$ that there is a point $z_{i j k}$ such that $\alpha_{i j k} \cap \mathrm{Bd} L_{i j}=\left\{z_{i j k}\right\}$; further, $z_{i j k} \in \operatorname{Int} B_{i j}$ and $z_{i j k} \notin W_{i j}$. Similarly, there is a point $z_{i j k}$ such that $z_{i j k} \in \operatorname{Int} B_{i k}, z_{i k j} \notin W_{i k}$, and $\alpha_{i j k} \cap \operatorname{Bd} L_{i k}$ $=\left\{z_{i k j}\right\}$. There are points $z_{j i k}$ and $z_{j k i}$ of $\alpha_{j i k}$, and $z_{k i j}$ and $z_{k j i}$ of $\alpha_{k i j}$ having analogous properties.

It follows from the construction of the 2-sphere $S_{i j}$ (in Step 6) that $\rho_{i j k}$ has exactly two points, $\tilde{z}_{i j k}$ and $\tilde{z}_{j i k}$, in common with $S_{i j}$.

Let $\sigma$ be some 3-simplex of $T$ having $\left\langle v_{i} v_{j} v_{k}\right\rangle$ as a face. Then different components of $\rho_{i j k}-\left\{\tilde{z}_{i j k}, \tilde{z}_{j i k}\right\}$ lie in different components of $U_{\sigma}-S_{i j}$, and hence in different components of $M-S_{i j}$.

Since

$$
V_{i j k} \cup\left(\operatorname{Int} Y_{i j}\right) \cup\left(\operatorname{Int} Y_{i k}\right) \cup\left(\operatorname{Int} Y_{j k}\right)
$$

is simply connected, then by Lemma 1 ,

$$
P^{-1}\left[V_{i j k} \cup\left(\text { Int } Y_{i j}\right) \cup\left(\text { Int } Y_{i k}\right) \cup\left(\text { Int } Y_{j k}\right)\right]
$$

is simply connected. Hence $\rho_{i j k}$ bounds a polyhedral singular disc $\rho_{i j k}^{*}$ lying in

$$
P^{-1}\left[V_{i j k} \cup\left(\text { Int } Y_{i j}\right) \cup\left(\text { Int } Y_{i k}\right) \cup\left(\text { Int } Y_{j k}\right)\right] \text {, }
$$


in $U_{\sigma}$, and in general position relative to $S_{i j}$. Note that $\rho_{i j k}^{*}$ is disjoint from $\tilde{\Delta}_{i j}$ and $\tilde{\Delta}_{j i}$.

By Lemma 4, there is an arc $\tilde{z}_{i j k} \tilde{z}_{j i k}$ on $S_{i j}$ from $\tilde{z}_{i j k}$ to $\tilde{z}_{j i k}$ and contained in $S_{i j} \cap \rho_{i j k}^{*}$. Since $S_{i j}$ is disjoint from

$$
P^{-1}\left[\left(\operatorname{Int} Y_{i j}\right) \cup\left(\text { Int } Y_{i k}\right) \cup\left(\operatorname{Int} Y_{j k}\right)\right]
$$

by construction, it follows that $\tilde{z}_{i j k} \tilde{z}_{j i k}$ lies in $\tilde{V}_{i j k}$. Further, $\tilde{z}_{i j k} \tilde{z}_{j k k} \subset$ Int $A_{i j}^{\prime}$.

Recall that $A_{i j} \cap\left\langle v_{i} v_{j} v_{k}\right\rangle$ is an arc $\pi_{i j k}$. Let $q_{i j k}$ and $q_{j i k}$ denote the endpoints of $\pi_{i j k}$ on Bd $C_{i}$ and Bd $C_{j}$, respectively. Clearly $z_{i j k}$ and $z_{j t k}$ belong to Int $\pi_{i j k}$. It follows that the subarcs $q_{i j k} z_{i j k}$ and $q_{j k k} z_{j i k}$ of $\pi_{i j k}$ are disjoint from $\mathrm{Cl} P\left[H_{G}\right]$.

Let $t_{i j k}$ be the last point on $\tilde{z}_{i j k} \tilde{z}_{j i k}$ (in the order from $\tilde{z}_{i j k}$ to $\tilde{z}_{j i k}$ on $\tilde{z}_{i j k} \tilde{z}_{j t k}$ ) of $\tilde{q}_{i j k} \tilde{z}_{i j k}$, and let $t_{j i k}$ be the first point on $\tilde{z}_{j k i} \tilde{q}_{j t k}$ (in the order from $\tilde{z}_{j i k}$ to $\tilde{q}_{j i k}$ on $\tilde{z}_{j i k} \tilde{q}_{j i k}$ ) of the subarc $t_{i j k} \tilde{z}_{j i k}$ of $\tilde{z}_{i j k} \tilde{z}_{j i k}$. Then let $\pi_{i j k}^{\prime}$ denote the union of

(1) the subarc $\tilde{q}_{i j k} t_{i j k}$ of $\tilde{q}_{i j k} \tilde{z}_{i j k}$,

(2) the subarc $t_{i j k} t_{j i k}$ of $\tilde{z}_{i j k} \tilde{z}_{j k k}$, and

(3) the subarc $t_{j i k} \tilde{q}_{j i k}$ of $\tilde{z}_{j i k} \tilde{q}_{j i k}$.

Consider the disc $m_{i j k}$. Since $V_{i j k} \cap m_{i j k}$ is connected, there is an arc $\beta_{i j k}$ spanning $\tilde{m}_{i j k}$, having endpoints $t_{i j k}$ and $t_{i k j}$, and lying in $\tilde{V}_{i j k}$. Let $n_{i j k}$ be the subdisc of $m_{i j k}$ which is the closure of the component of $m_{i j k}-P\left[\beta_{i j k}\right]$ containing points on $\mathrm{Bd} C_{i}$. Then $n_{i j k}$ is disjoint from $\mathrm{Cl} P\left[H_{G}\right]$ because $m_{i j k}$ is disjoint from $\mathrm{Cl} P\left[H_{G}\right]$.

There exist arcs $\beta_{i j k}$ and $\beta_{k i j}$, and discs $n_{j t k}$ and $n_{k i j}$ with analogous properties.

Let $\pi_{i j k}^{\prime \prime}$ denote the subarc $t_{i j k} t_{j i k}$ of $\pi_{i j k}^{\prime}$, and let $\pi_{j k i}^{\prime \prime}$ and $\pi_{i k j}^{\prime \prime}$ denote subarcs of $\pi_{j k i}^{\prime}$ and $\pi_{i k j}^{\prime}$, respectively, defined analogously.

Let $\mu_{i j k}$ denote

$$
\beta_{i j k} \cup \beta_{j i k} \cup \beta_{k i j} \cup \pi_{i j k}^{\prime \prime} \cup \pi_{j k i}^{\prime \prime} \cup \pi_{i k j}^{\prime \prime} .
$$

Then $\mu_{i j k}$ is a polygonal simple closed curve lying in $\tilde{V}_{i j k}$.

Step 9. In this step, we shall describe $\Sigma_{0}$ and $\Sigma_{1}$, and begin the construction of $\Sigma_{2} . \Sigma_{0}$ is defined to be

$$
\left\{\tilde{v}_{i}: v_{i} \text { is a vertex of } T\right\} .
$$

Suppose that $\left\langle v_{i} v_{j}\right\rangle$ is a 1 -simplex of $T$. Recall that $\left\langle v_{i} v_{j} v_{k_{1}}\right\rangle,\left\langle v_{i} v_{j} v_{k_{2}}\right\rangle, \ldots$, and $\left\langle v_{i} v_{j} v_{\left.k_{d(1)}\right)}\right\rangle$ are the 2-simplexes of $T$ having $\left\langle v_{i} v_{j}\right\rangle$ as an edge. If $s=1,2, \ldots$, or $d(i j)$, there is an $\operatorname{arc} \pi_{i j k_{s}}^{\prime}$ with one endpoint on Bd $\tilde{\Delta}_{i j}$, the other on Bd $\tilde{\Delta}_{j i}$, spanning $A_{i j}^{\prime}$, and lying in $\tilde{V}_{i j k_{s}}$; see Step 8.

There is a piecewise linear homeomorphism $g_{i j}$ from $L_{i j}$ onto $L_{i j}^{\prime}$ such that (1) $g_{i j}\left|\left(\Delta_{i} \cup \Delta_{j}\right)=P^{-1}\right|\left(\Delta_{i} \cup \Delta_{f}\right)$ and (2) if $k=1,2, \ldots$, or $d(i j), g_{i j}\left[\pi_{i j k}\right]=\pi_{i j k}^{\prime}$. Now let $g$ be the piecewise linear homeomorphism from $K$ onto $K^{\prime}$ defined as follows:

(1) If $v_{i}$ is a vertex of $T, g\left|C_{i}=P^{-1}\right| C_{t}$.

(2) If $\left\langle v_{i} v_{j}\right\rangle$ is a 1 -simplex of $T, g \mid L_{i j}=g_{i j}$. 
For each 1-simplex $\left\langle v_{i} v_{j}\right\rangle$ of $T$, let $e_{i j}$ denote $g\left[\left\langle v_{i} v_{j}\right\rangle\right]$. Let $\Sigma_{1}$ denote

$$
\bigcup\left\{e_{i j}:\left\langle v_{i} v_{j}\right\rangle \text { is a 1-simplex of } T\right\} \text {. }
$$

Step 10. Suppose that $\left\langle v_{i} v_{j} v_{k}\right\rangle$ is a 2-simplex of $T$. Corresponding to $\left\langle v_{i} v_{j} v_{k}\right\rangle$, there is to be a 2-simplex $D_{i j k}$ of $\Sigma$. The boundary of $D_{i j k}$ is to be $\gamma_{i j k}$ where $\gamma_{i j k}$ $=g\left[\mathrm{Bd}\left\langle v_{i} v_{j} v_{k}\right\rangle\right]$. We get $D_{i j k}$ by constructing two sets as follows: (1) A nonsingular annulus $O_{i j k}$ with boundary curves $\gamma_{i j k}$ and $\mu_{i j k}$ (described in Step 8) and (2) a singular disc $R_{i j k}$ with boundary $\mu_{i j k}$ and disjoint from $\gamma_{i j k}$. In this step, we describe $O_{i j k}$ and a certain singular disc $R_{i j k}^{0}$ to be used in the construction of $R_{i j k}$.

First we shall describe the annulus $O_{i j k}$. Let $\gamma_{i j k}$ denote $g\left[\mathrm{Bd}\left\langle v_{i} v_{j} v_{k}\right\rangle\right] ; \gamma_{i j k}$ $=e_{i j} \cup e_{i k} \cup e_{j k}$. Let $O_{i j k}$ denote

$$
g\left[K \cap\left\langle v_{i} v_{j} v_{k}\right\rangle\right] \cup n_{i j k} \cup n_{j i k} \cup n_{k j i} ;
$$

the discs $n_{i j k}, n_{j i k}$, and $n_{k i j}$ are described in Step 8. Clearly $O_{i j k}$ is a polyhedral annulus and $\mathrm{Bd} O_{i j k}=\gamma_{i j k} \cup \mu_{i j k}$. Further, if $\left\langle v_{s} v_{t} v_{u}\right\rangle$ is a 2-simplex of $T$ distinct from $\left\langle v_{i} v_{j} v_{k}\right\rangle$, then $O_{i j k}$ and $O_{s t u}$ intersect if and only if $\left\langle v_{i} v_{j} v_{k}\right\rangle$ and $\left\langle v_{s} v_{t} v_{u}\right\rangle$ intersect, and (1) if $\left\langle v_{t} v_{j} v_{k}\right\rangle \cap\left\langle v_{s} v_{t} v_{u}\right\rangle$ is a 1-simplex $\left\langle v_{l} v_{r}\right\rangle$ of $T$, then $O_{i j k} \cap O_{s t u}=e_{l r}$, and (2) if, for some vertex $v_{l}$ of $T,\left\langle v_{i} v_{j} v_{k}\right\rangle \cap\left\langle v_{s} v_{t} v_{u}\right\rangle=\left\{v_{l}\right\}$, then $O_{i j k} \cap O_{s t u}=\left\{\tilde{v}_{l}\right\}$.

We shall now construct the singular disc $R_{i j k}$. We first construct strips to be attached to $A_{i j}^{\prime}, A_{i k}^{\prime}$, and $A_{j k}^{\prime}$; the purpose of these strips is to insure that $R_{i j k}$ is in normal position relative to each of $L_{i j}^{\prime}, L_{i k}^{\prime}$, and $L_{j k}^{\prime}$. Let $s_{i j k}^{0}$ be a disc lying in $\tilde{V}_{i j k}$ whose boundary is the union of the following arcs:

(1) The arc $\mu_{i j k} \cap A_{i j}^{\prime}$.

(2) A very short subarc $t_{i j k} t_{i j k}^{\prime}$ of the arc $t_{i j k} t_{i k j}$.

(3) A very short subarc $t_{i j k} t_{j i k}^{\prime}$ of the arc $t_{j i k} t_{j k i}$.

(4) An arc $\beta_{i j k}^{*}$ from $t_{i j k}^{\prime}$ to $t_{j i k}^{\prime}$, lying in $\tilde{V}_{i j k}$, disjoint from

$$
\tilde{C}_{i} \cup \tilde{C}_{j} \cup \tilde{C}_{k} \cup L_{i j}^{\prime} \cup L_{i k}^{\prime} \cup L_{j k}^{\prime},
$$

and "parallel to" the arc $\mu_{i j k} \cap A_{i j}^{\prime}$. We think of $s_{i j k}^{0}$ as a narrow strip attached to $A_{i j}^{\prime}$.

There exist strips $s_{j i k}^{0}$ and $s_{k i j}^{0}$, points $t_{j k i}^{\prime}$ of the arc $t_{j i k} t_{j k i}, t_{k i j}^{\prime}$ and $t_{k j i}^{\prime}$ of the arc $t_{k i j} t_{k j i}$, and $t_{i k j}^{\prime}$ of the $\operatorname{arc} t_{i j k} t_{i k j}$, and $\operatorname{arcs} \beta_{j k i}^{*}$ from $t_{j k i}^{\prime}$ to $t_{k j i}^{\prime}$, and $\beta_{k i j}^{*}$ from $t_{k i j}$ to $t_{k j i}$, having analogous properties.

Let $\mu_{i j k}^{\prime}$ denote the simple closed curve

$$
\beta_{i j k}^{*} \cup \beta_{j i k}^{*} \cup \beta_{k i j}^{*} \cup t_{i j k}^{\prime} t_{i k j}^{\prime} \cup t_{j i k}^{\prime} t_{j k i}^{\prime} \cup t_{k i j}^{\prime} t_{k j i}^{\prime}
$$

$\mu_{i j k}^{\prime}$ lies in $\tilde{V}_{i j k}$. Since $\tilde{V}_{i j k}$ is simply connected, there is a polyhedral singular disc $R_{i j k}^{\prime}$ such that $\mathrm{Bd} R_{i j k}^{\prime}=\mu_{i j k}^{\prime}$ and $R_{i j k}^{\prime} \subset \tilde{V}_{i j k}$. Let $R_{i j k}^{0}$ denote

$$
R_{i j k}^{\prime} \cup s_{i j k}^{0} \cup s_{j i k}^{0} \cup s_{k i j}^{0}
$$


$R_{i j k}^{0}$ is a polyhedral singular disc lying in $\tilde{V}_{i j k}$ and such that (1) Bd $R_{i j k}^{0}=\mu_{i j k}$ and (2) $R_{i j k}^{0}$ is in normal position relative to each of $L_{i j}^{\prime}, L_{i k}^{\prime}$ and $L_{j k}^{\prime}$.

Note that if $\left\langle v_{i} v_{j} v_{k}\right\rangle$ and $\left\langle v_{s} v_{t} v_{u}\right\rangle$ are distinct 2-simplexes of $T$, then $R_{i j k}^{0}$ and $R_{s t u}^{0}$ are disjoint.

Step 11. It may not be true that for each 1-simplex $\left\langle v_{i} v_{j} v_{k}\right\rangle$ of $T, R_{i j k}^{0}$ and $\gamma_{i j k}$ are disjoint. In this step, we use Lemma 5 to obtain mutually disjoint singular discs $R_{i j k}$, each disjoint from $\Sigma_{1}$.

Consider the 1-simplexes $\left\langle v_{i_{1}} v_{j_{1}}\right\rangle,\left\langle v_{i_{2}} v_{j_{2}}\right\rangle, \ldots$ of $T$. Let $U_{1}^{\prime}, U_{2}^{\prime}, \ldots$ be mutually disjoint open sets in $M$ such that if $s=1,2, \ldots$, then

(1) Int $A_{i_{s} s_{s}}^{\prime} \subset U_{s}^{\prime}$,

(2) for each vertex $v_{t}$ of $T, U_{s}^{\prime}$ is disjoint from $\tilde{C}_{t}$,

(3) if $\left\langle v_{i} v_{j} v_{k}\right\rangle$ is a 2-simplex of $T$, then $R_{i j k}^{0}$ intersects $U_{s}^{\prime}$ if and only if $R_{i j k}^{0}$ intersects $A_{i_{s} j_{s}}^{\prime}$, and

(4) if $\sigma$ is any 3-simplex of $T$ having $\left\langle v_{i_{s}} v_{j_{s}}\right\rangle$ as an edge, then $U_{s}^{\prime} \subset U_{\sigma}$.

Recall that if $\left\langle v_{i_{t}} v_{j_{t}}\right\rangle$ is a 1-simplex of $T$, there are $d\left(i_{t} j_{t}\right)$ distinct 2-simplexes of $T$ having $\left\langle v_{i_{t}} v_{j_{t}}\right\rangle$ as an edge. If $s=1,2, \ldots$, or $d\left(i_{t} j_{t}\right)$ and $\left\langle v_{i_{t}} v_{j_{t}} v_{k_{s}}\right\rangle$ is a 2-simplex of $T$, let $\boldsymbol{R}_{t s}^{0}$ denote $\boldsymbol{R}_{\boldsymbol{i}_{t} j_{t} \boldsymbol{k}_{s}}^{0}$.

First consider $\left\langle v_{i_{1}} v_{j_{1}}\right\rangle$. By Lemma 5 , there exist mutually disjoint polyhedral singular discs $R_{11}^{1}, R_{12}^{1}, \ldots$, and $R_{1 d\left(i_{1} j_{1}\right)}^{1}$ such that if $s=1,2, \ldots$, or $d\left(i_{1} j_{1}\right)$, then

(1) $R_{1 s}^{1} \cap L_{i_{1} j_{1}}^{\prime}=\pi_{i_{1} j_{1} k_{1 s}}^{\prime}$,

(2) $\mathrm{Bd} R_{1 s}^{1}=\mathrm{Bd} R_{1 s}^{0}$, and

(3) $R_{1 s}^{1} \subset U_{1}^{\prime} \cup R_{1 s}^{0}$.

If $t=2,3, \ldots$, then for each 2-simplex $\left\langle v_{i_{t}} v_{s_{t}} v_{k_{s}}\right\rangle$ of $T$, let $R_{t s}^{1}$ denote $R_{t s}^{0}$. Then the following statements hold:

(1) If $\left\langle v_{i_{r}} v_{j_{r}} v_{k_{s}}\right\rangle$ and $\left\langle v_{i_{t}} v_{j_{t}} v_{k_{u}}\right\rangle$ are distinct 2-simplexes of $T$, then $R_{r s}^{1}$ and $R_{t u}^{1}$ are disjoint.

(2) If $\left\langle v_{t_{r}} v_{r_{r}} v_{k_{s}}\right\rangle$ is a 2-simplex of $T$ and $v_{t}$ is a vertex of $T$, then $R_{r s}^{1}$ and $\widetilde{C}_{t}$ are disjoint.

(3) If $\left\langle v_{i_{r}} v_{j_{r}}\right\rangle$ and $\left\langle v_{t} v_{u}\right\rangle$ are 1-simplexes of $T$ distinct from $\left\langle v_{i_{1}} v_{j_{1}}\right\rangle$ and $s$ is a positive integer such that $\left\langle v_{i_{r}} v_{j_{r}} v_{k_{s}}\right\rangle$ is a 2-simplex of $T$, then $R_{r s}^{1} \cap S_{t u}=$ $R_{r s}^{0} \cap S_{t u}$.

Now consider $\left\langle v_{i_{2}} v_{j_{2}}\right\rangle$. The process described above, which used $L_{i_{1} j_{1}}^{\prime}$ and the singular discs $R_{11}^{0}, R_{12}^{0}, \ldots$, and $R_{1 d\left(i_{1} j_{1}\right)}^{0}$, may be repeated using $L_{i_{2} j_{2}}^{\prime}$ and the singular discs $R_{21}^{1}, R_{22}^{1}, \ldots$, and $R_{2 d\left(i_{2} j_{2}\right)}^{1}$. There result mutually disjoint polyhedral singular discs $R_{21}^{2}, R_{22}^{2}, \ldots$, and $R_{2 d\left(i_{2} j_{2}\right)}^{2}$. If $t=1,3,4, \ldots$, then for each 2-simplex $\left\langle v_{i_{t}} v_{j_{t}} v_{k_{s}}\right\rangle$ of $T$, let $R_{t s}^{2}$ denote $R_{t s}^{1}$. The singular discs $R_{t s}^{2}$ have properties analogous to those of the discs $R_{t s}^{1}$ mentioned above, and, in addition, the following: For any 2-simplex $\left\langle v_{i_{r}} v_{j_{r}} v_{k_{s}}\right\rangle$ of $T$, if $u=1$ or 2 and $S_{i_{u} j_{u}}$ and $R_{r s}^{2}$ intersect, then $i_{u}=i_{r}$, $j_{u}=j_{r}$, and $S_{i_{u} j_{u}} \cap R_{r s}^{2}=\pi_{i_{r} j_{r} k_{s}}^{\prime}$.

This process may be continued, considering $\left\langle v_{i_{3}} v_{j_{3}}\right\rangle,\left\langle v_{i_{4}} v_{j_{4}}\right\rangle, \ldots$ There results, for each 2-simplex $\left\langle v_{i} v_{j} v_{k}\right\rangle$ of $T$, a polyhedral singular disc $R_{i j k}$ such that the following statements hold: 
(1) For any 2-simplex $\left\langle v_{i} v_{j} v_{k}\right\rangle$ of $T, \mathrm{Bd} R_{i j k}=\mathrm{Bd} R_{i j k}^{0}$, and for each vertex $v_{t}$ of $T, R_{i j k}$ and $\tilde{C}_{t}$ are disjoint.

(2) If $\left\langle v_{i} v_{j} v_{k}\right\rangle$ and $\left\langle v_{s} v_{t} v_{u}\right\rangle$ are distinct 2-simplexes of $T$, then $R_{i j k}$ and $R_{s t u}$ are disjoint.

(3) If $\left\langle v_{i} v_{j}\right\rangle$ is any 1-simplex of $T$ and $\left\langle v_{s} v_{t} v_{u}\right\rangle$ is any 2-simplex of $T$, then $L_{i j}^{\prime}$ and $R_{\text {stu }}$ intersect if and only if $i$ and $j$ belong to $\{s, t, u\}$ and if $R_{\text {stu }}$ intersects $L_{i j}^{\prime}$, $R_{s t u} \cap L_{i j}^{\prime}$ is the arc $\pi_{s t u}^{\prime}$.

Now we may construct the carrier $\Sigma_{2}$ of the 2-skeleton of $\Sigma$. Suppose that $\left\langle v_{i} v_{j} v_{k}\right\rangle$ is a 2-simplex of $T$. It is clear that $R_{i j k}$ does not intersect

$$
\text { Int }\left[\tilde{C}_{i} \cup \tilde{C}_{j} \cup \tilde{C}_{k} \cup L_{i j}^{\prime} \cup L_{i k}^{\prime} \cup L_{j k}^{\prime}\right] \text {. }
$$

Hence $R_{i j k} \cup O_{i j k}$ is a polyhedral singular disc with boundary $\gamma_{i j k}$, and there is a neighborhood of $\gamma_{i j k}$ containing no point of singularity of $R_{i j k} \cup O_{i j k}$. Further, if $\sigma$ is any 3-simplex of $T$ having $\left\langle v_{i} v_{j} v_{k}\right\rangle$ as a face, then $R_{i j k} \cup O_{i j k} \subset U_{\sigma}$.

With the aid of Dehn's lemma as proved by Papakyriakopoulos [11], it may be shown that for each 2-simplex $\left\langle v_{i} v_{j} v_{k}\right\rangle$ of $T$, there is a polyhedral disc $D_{i j k}$ such that

(1) $\operatorname{Bd} D_{i j k}=\gamma_{i j k}$,

(2) there is an annulus on $D_{i j k} \cap O_{i j k}$ having $\gamma_{i j k}$ as one boundary component,

(3) if $\left\langle v_{s} v_{t} v_{u}\right\rangle$ is any 2-simplex of $T$ distinct from $\left\langle v_{i} v_{j} v_{k}\right\rangle$, then $D_{i j k} \cap D_{s t u}$ $=O_{i j k} \cap O_{s t u}$, and

(4) if $\sigma$ is any 3-simplex of $T$ having $\left\langle v_{i} v_{j} v_{k}\right\rangle$ as a face, then $D_{i j k} \subset U_{\sigma}$.

Define $\Sigma_{2}$ to be

$$
\bigcup\left\{D_{i j k}:\left\langle v_{i} v_{j} v_{k}\right\rangle \text { is a 2-simplex of } T\right\} \text {. }
$$

Step 12. We may now define $\Sigma$ and construct an embedding $h$ of $T_{2}$ into $M$. Define $\Sigma$ to be

$\left\{\tilde{v}_{i}: v_{i}\right.$ is a vertex of $\left.T\right\} \cup\left\{e_{i j}:\left\langle v_{i} v_{j}\right\rangle\right.$ is a 1-simplex of $\left.T\right\}$

$$
\cup\left\{D_{i j k}:\left\langle v_{i} v_{j} v_{k}\right\rangle \text { is a 2-simplex of } T\right\} \text {. }
$$

Let $\varphi$ be the function from the 2-skeleton of $T$ onto $\Sigma$ defined as follows: (1) If $v_{i}$ is a vertex of $T, \varphi\left(v_{i}\right)=\tilde{v}_{i}$. (2) If $\left\langle v_{i} v_{j}\right\rangle$ is a 1-simplex of $T, \varphi\left(\left\langle v_{i} v_{j}\right\rangle\right)=e_{i j}$. (3) If $\left\langle v_{i} v_{j} v_{k}\right\rangle$ is a 2-simplex of $T, \varphi\left(\left\langle v_{i} v_{j} v_{k}\right\rangle\right)=D_{i j k}$. Clearly $\varphi$ is an isomorphism.

$\Sigma$ is a locally finite collection of subsets of $M$. This is true because (1) $\left\{U_{\sigma}: \sigma\right.$ is a 3-simplex of $T\}$ is locally finite and (2) if $\sigma$ is any 3-simplex of $T$ and $\tau$ is any proper face of $\sigma, \varphi(\tau) \subset U_{\sigma}$.

It follows that there is a piecewise linear homeomorphism $h$ from $T_{2}$ onto $\Sigma_{2}$ such that (1) for each vertex $v_{i}$ of $T, h\left(v_{i}\right)=\tilde{v}_{i}$, (2) for each 1-simplex $\left\langle v_{i} v_{j}\right\rangle$ of $T$, $h\left[\left\langle v_{i} v_{j}\right\rangle\right]=e_{i j}$, and (3) for each 2-simplex $\left\langle v_{i} v_{j} v_{k}\right\rangle$ of $T, h\left[\left\langle v_{i} v_{j} v_{k}\right\rangle\right]=D_{i j k}$. It is clear that if $\sigma$ is any 3-simplex of $T, h[\mathrm{Bd} \sigma] \subset U_{\sigma}$. Recall that the set $X_{\sigma}$ is constructed in Step 1 so that if $\tau$ is a 3-simplex of $T$ distinct from $\sigma, X_{\sigma}$ contains no vertex of $\tau$. It follows that if $\tau$ is any 3-simplex of $T$ distinct from $\sigma, h[\mathrm{Bd} \tau]$ does not lie in $U_{\sigma}$. 
In addition, $U_{\sigma} \neq U_{\imath}$. Then by Lemma $8, M$ and $N$ are homeomorphic. This completes the proof of the theorem.

\section{Corollaries of the main result.}

Corollary 1. Suppose $M$ is a 3-manifold, $G$ is a cellular decomposition of $M$ such that $M / G$ is a 3-manifold $N$, and $N$ has a triangulation $T$ such that (1) no vertex of $T$ belongs to $\mathrm{Cl} P\left[H_{G}\right]$ and (2) if $\sigma$ is any 3-simplex of $T$, there is an open 3-cell in $M$ containing $P^{-1}[\sigma]$. Then $M$ and $N$ are homeomorphic.

Proof. Apply the theorem to each component of $M$.

Corollary 2. Suppose that $M$ is a 3-manifold, $G$ is a cellular decomposition of $M$ such that $M / G$ is a 3-manifold $N$, and $P\left[H_{G}\right]$ is a nowhere dense subset of $N$. Then $M$ and $N$ are homeomorphic.

Proof. Since $G$ is a cellular decomposition of $M$, there is a triangulation $T^{0}$ of $N$ such that if $\sigma$ is any 3-simplex of $T^{0}, P^{-1}[\sigma]$ lies in some open 3-cell in $M$. Since $P\left[H_{G}\right]$ is nowhere dense in $N$, so is $\mathrm{Cl} P\left[H_{G}\right]$. A slight adjustment of the vertices of $T^{0}$ yields a triangulation $T$ of $N$ such that (1) no vertex of $T$ belongs to $\mathrm{Cl} P\left[H_{G}\right]$ and (2) if $\sigma$ is any 3-simplex of $T, P^{-1}[\sigma]$ lies in an open 3-cell in $M$. By Corollary 1 , $M$ and $N$ are homeomorphic.

Corollary 2 is a generalization of the result announced in [3].

COROLlaRY 3. If $M$ is a 3-manifold, $G$ is a cellular decomposition of $M$ such that $M / G$ is a 3-manifold $N$, and $P\left[H_{G}\right]$ lies in a closed set of dimension at most two, then $M$ and $N$ are homeomorphic.

The following corollary is a generalization of Theorem 3 of [2].

COROLlARY 4. If $M$ is a 3-manifold, $G$ is a cellular decomposition of $M, M / G$ is a 3-manifold $N$, and $P\left[H_{G}\right]$ is contained in a closed 0 -dimensional set, then $M$ and $N$ are homeomorphic.

The next two corollaries deal with point-like decompositions of $E^{3}$ and $S^{3}$. It is clear that if $M$ is either $E^{3}$ or $S^{3}, G$ is a point-like decomposition of $M$, and $\sigma$ is a compact proper subset of $M$, then $P^{-1}[\sigma]$ lies in an open 3-cell in $M$. It was mentioned in $\$ 2$ that in $E^{3}$ and $S^{3}$, "point-like" and "cellular" are equivalent.

Corollary 5. Suppose that $M$ is either $E^{3}$ or $S^{3}, G$ is a point-like decomposition of $M$, and $M / G$ is a 3-manifold $N$ such that $N$ has a triangulation, no vertex of which belongs to $\mathrm{Cl} P\left[H_{G}\right]$. Then $M$ and $N$ are homeomorphic.

Corollary 6. Suppose that $G$ is a point-like decomposition of $S^{3}, S^{3} / G$ is a 3-manifold $N$, and there is an open set $U$ such that $U$ and $P\left[H_{G}\right]$ are disjoint. Then $N$ is homeomorphic to $S^{3}$.

Proof. $N$ is a compact connected 3-manifold. Hence $N$ has a triangulation, each vertex of which lies in $U$. Then Corollary 6 follows from Corollary 5 . 
8. Cellular maps of compact 3-manifolds. In this section, we shall formulate some of the results of this paper in terms of continuous functions rather than upper semicontinuous decompositions. We restrict our attention to compact manifolds.

If $M$ is a 3-manifold, by a cellular map from $M$ into a space is meant a continuous function with domain $M$ such that if $y \in f[M], f^{-1}[y]$ is a cellular subset of $M$. If $f$ is a continuous function from a space $X$ onto a space $Y$, then a point $p$ of $Y$ is a nonsingular point of $f$ if and only if there is an open neighborhood $U$ of $p$ such that $f^{-1} \mid U$ is a homeomorphism. A point $p$ of $Y$ is a singular point of $f$ if and only if $p$ is not a nonsingular point of $f$. The set of all singular points of $f$ is closed in $Y$.

COROllaRY 7. Suppose that $f$ is a cellular map from a compact 3-manifold $M$ onto a 3-manifold $N$. Suppose $N$ has a triangulation $T$ such that (1) no vertex of $T$ is a singular point of $f$ and (2) if $\sigma$ is any 3-simplex of $T$, then $f^{-1}[\sigma]$ lies in an open 3-cell in $M$. Then $M$ and $N$ are homeomorphic.

COROLlaRY 8. Suppose that $f$ is a cellular map from a compact 3-manifold $M$ onto a 3-manifold $N$ such that the set of all singular points of $f$ is nowhere dense in $N$. Then $M$ and $N$ are homeomorphic.

COROLlaRY 9. If $f$ is a cellular map from $S^{3}$ onto a 3-manifold $N$ and the set of all singular points of $f$ is a proper subset of $N$, then $N$ is homeomorphic to $S^{3}$.

\section{REFERENCES}

1. S. Armentrout, Upper semicontinuous decompositions of $E^{3}$ with at most countably many nondegenerate elements, Ann. of Math. 78 (1963), 605-618.

2. - Decompositions of $E^{3}$ with a compact 0-dimensional set of nondegenerate elements, Trans. Amer. Math. Soc. 123 (1966), 165-177.

3. - Concerning point-like decompositions of $S^{3}$ that yield 3-manifolds, Notices Amer. Math. Soc. 12 (1965), 90.

4. R. H. Bing, Point-like decompositions of $E^{3}$, Fund. Math. 50 (1962), 431-453.

5. - An alternative proof that 3-manifolds can be triangulated, Ann. of Math. 69 (1959), $37-65$.

6. - Approximating surfaces with polyhedral ones, Ann. of Math. 65 (1957), 456-483.

7. - Locally tame sets are tame, Ann. of Math. 59 (1954), 145-158.

8. R. Finney, Point-like, simplicial mappings of a 3-sphere, Canad. J. Math. 15 (1963), 591-604.

9. E. E. Moise, Affine structures in 3-manifolds, V: The triangulation theorem and Hauptvermutung, Ann. of Math. 56 (1952), 92-114.

10. R. L. Moore, Foundations of point set theory, rev. ed., Amer. Math. Soc. Colloq. Publ., Vol. 13, Amer. Math. Soc., Providence, R. I., 1962.

11. C. D. Papakyriakopoulos, On Dehn's lemma and the asphericity of knots, Ann. of Math. 66 (1957), 1-26.

12. T. M. Price, Cellular decompositions of $E^{3}$, Thesis, University of Wisconsin, Madison, 1964.

13. - A necessary condition that a cellular upper semicontinuous decomposition of $E^{n}$ yield $E^{n}$, Trans. Amer. Math. Soc. 122 (1966), 427-435.

14. A. Shapiro and J. H. C. Whitehead, A proof and extension of Dehn's lemma, Bull. Amer. Math. Soc. 64 (1958), 174-178. 
15. D. G. Stewart, Cellular subsets of the 3-sphere, Trans. Amer. Math. Soc. 114 (1965), 10-22.

16. J. H. C. Whitehead, Simplicial spaces, nuclei, and m-groups, Proc. London Math. Soc. 45 (1939), 243-327.

17. G. T. Whyburn, Analytic topology, Amer. Math. Soc. Colloq. Publ., Vol. 28, Amer. Math. Soc., Providence, R. I., 1942.

THE UNIVERSITY OF IOWA, Iowa CrTy, IOWA

The Institute for Advanced Study, Princeton, New Jersey

The UnIVERSITY OF Wisconsin, MADISON, Wisconsin 